Article

\title{
Expert Hypertension Detection System Featuring Pulse Plethysmograph Signals and Hybrid Feature Selection and Reduction Scheme
}

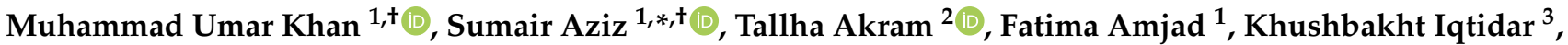 \\ Yunyoung Nam ${ }^{4, *}$ and Muhammad Attique Khan ${ }^{5}$
}

1 Department of Electronics Engineering, University of Engineering and Technology Taxila, Taxila 47050, Pakistan; sa.umarkhan@gmail.com (M.U.K.); fatimaamjad1997@gmail.com (F.A.)

2 Department of Electrical and Computer Engineering, COMSATS University Islamabad, Wah Campus, Wah Cantonment, Islamabad 45550, Pakistan; tallha@ciitwah.edu.pk

3 Department of Computer and Software Engineering, College of Electrical and Mechanical Engineering, National University of Sciences and Technology, Islamabad 44000, Pakistan; khushbakht.iqtidar18@ce.ceme.edu.pk

4 Department of Computer Science and Engineering, Soonchunhyang University, Asan 31538, Korea

5 Department of Computer Science, HITEC University, Taxila 47080, Pakistan; attique.khan@hitecuni.edu.pk

* Correspondence: sumair.aziz@uettaxila.edu.pk (S.A.); ynam@sch.ac.kr (Y.N.)

+ These authors contributed equally to this work.

Citation: Khan, M.U.; Aziz, S.; Akram, T.; Amjad, F.; Iqtidar, K.; Nam, Y.; Khan, M.A. Expert Hypertension Detection System Featuring Pulse Plethysmograph Signals and Hybrid Feature Selection and Reduction Scheme. Sensors 2021, 21, 247. https://dx.doi.org/10.3390/s21010247

Received: 1 December 2020 Accepted: 24 December 2020 Published: 2 January 2021

Publisher's Note: MDPI stays neutral with regard to jurisdictional clai$\mathrm{ms}$ in published maps and institutional affiliations.

Copyright: (C) 2021 by the authors. Licensee MDPI, Basel, Switzerland. This article is an open access article distributed under the terms and conditions of the Creative Commons Attribution (CC BY) license (https:// creativecommons.org/licenses/by/ $4.0 /)$.

\begin{abstract}
Hypertension is an antecedent to cardiac disorders. According to the World Health Organization (WHO), the number of people affected with hypertension will reach around 1.56 billion by 2025. Early detection of hypertension is imperative to prevent the complications caused by cardiac abnormalities. Hypertension usually possesses no apparent detectable symptoms; hence, the control rate is significantly low. Computer-aided diagnosis based on machine learning and signal analysis has recently been applied to identify biomarkers for the accurate prediction of hypertension. This research proposes a new expert hypertension detection system (EHDS) from pulse plethysmograph (PuPG) signals for the categorization of normal and hypertension. The PuPG signal data set, including rich information of cardiac activity, was acquired from healthy and hypertensive subjects. The raw PuPG signals were preprocessed through empirical mode decomposition (EMD) by decomposing a signal into its constituent components. A combination of multi-domain features was extracted from the preprocessed PuPG signal. The features exhibiting high discriminative characteristics were selected and reduced through a proposed hybrid feature selection and reduction (HFSR) scheme. Selected features were subjected to various classification methods in a comparative fashion in which the best performance of $99.4 \%$ accuracy, $99.6 \%$ sensitivity, and $99.2 \%$ specificity was achieved through weighted $k$-nearest neighbor $(\mathrm{KNN}-\mathrm{W})$. The performance of the proposed EHDS was thoroughly assessed by tenfold cross-validation. The proposed EHDS achieved better detection performance in comparison to other electrocardiogram (ECG) and photoplethysmograph (PPG)-based methods.
\end{abstract}

Keywords: pulse plethysmograph; biomedical signal processing; feature extraction; machine learning; feature selection and reduction; empirical mode decomposition; discrete wavelet transform; hypertension

\section{Introduction}

Hypertension, also known as high blood pressure, is one of the most common risk factor for cardiovascular disease (CVD) [1]. It is a very common condition in which a large amount of force from the blood pushes on the walls of the arteries leading towards heart diseases [2]. The main risk factors for hypertension include age, genetics, gender, lack of physical activity, bad diet practices, high cholesterol, excessive salt consumption, less intake of vegetables and fruit, smoking, obesity, family history, and other diseases such 
as kidney disease or diabetes [3]. According to the World Health Organization (WHO) statistics, 1.13 million of the world population suffers from hypertension, and more men are affected than women. One out of every four men suffers from high blood pressure issues [3]. It is a silent killer that affects the most significant tissues of the human body [4]. Indeed, many people are not aware they have hypertension [5]. In the US, an estimated 13 million people are unaware of their condition [6], while in China, 59\% of people with hypertension are unaware of their condition [5]. In Pakistan, 18\% of the adults are affected by hypertension, and $33 \%$ of the adults above the age of 45 were affected according to the National Health Survey Pakistan [7]. Prevalence rates of hypertension based on genetic and ethnic variations ranges from about $29 \%$ for Asians, $45 \%$ for black men, and around $46.3 \%$ for women [8].

Table 1 describes a blood pressure ranges of normal and hypertension in terms of systolic and diastolic pressures. Some of the common symptoms of hypertension include headaches, dizziness, migraine, lightheadedness, changes in vision, or fainting episodes [9]. Hypertension serves as the first step towards CVDs, but the most chronic effect of unchecked hypertension is stroke, which can lead to permanent paralysis of certain body parts. Prolonged and undetected hypertension can be fatal; therefore, its detection in the preliminary stages is crucial.

Table 1. Categorization of blood pressure.

\begin{tabular}{ccc}
\hline Class & Systolic $(\mathbf{m m H g})$ & Diastolic $(\mathbf{m m H g})$ \\
\hline Optimal & Less than 120 & Less than 80 \\
Normal & 120 to 129 & 80 to 84 \\
High Normal & 130 to 139 & 85 to 89 \\
Hypertension & More than or equal to 140 & More than or equal to 90 \\
\hline
\end{tabular}

Moreover, the world is currently suffering from the outbreak of a pandemic COVID19 caused by the coronavirus SARS-CoV-2. It was reported that there are some specific comorbidities associated with a high risk of infection and increased severity of lung injury. Most of the common comorbidities in COVID-19 patients are hypertension (30\%), cardiovascular disease ( $8 \%)$, and diabetes (19\%) [10]. Therefore, it is not entirely surprising that the COVID-19 patients experiencing worst complications are hypertensive since hypertension is most frequent in older people and these elderlies are particularly at risk of being infected by a coronavirus [11]. Given the above information and statistics, it is clear that we need a technique for recognizing hypertension as early as possible to avoid significant damage to one's body.

Various techniques including physiological signals such as electrocardiogram (ECG) and photoplethymograph (PPG) are currently being used to detect hypertension. Detection of hypertension from PPG signals (MIMIC database) using continuous wavelet transform (CWT) and the GoogLeNet deep learning model [12] achieved an F1 score of $92.55 \%$. This work relies on a deep learning model so it requires high processing power, large scale data sets, and more training time. The authors of [13] proposed a method based on pulse arrival time (PAT) features extracted from PPG and ECG signals. The $k$-nearest neighbor $(\mathrm{KNN})$ classification method was employed to predict hypertension with an F1 score of $94.84 \%$. The research achieved acceptable results but missing consideration of preprocessing the PPG signals as PPG suffers from motion artifacts and variation in light intensity. Identification of hypertension [14] from heart rate variability (HRV) signals yielded an accuracy of $85.47 \%$ using standard deviation of all NN intervals and multiple instance learning (MIL). However, HRV feature extraction for long-term data requires significant processing resources. A model [15] to detect hypertension obtained $93.33 \%$ accuracy using Savitzky-Golay filtering (SGF), entropy features extracted from ECG, and a support vector machine (SVM) classifier. The method achieved a considerable performance on a comparatively small data set consisting of 48 participants. 
In [16], the authors proposed a hypertension detection framework based on five principal components extracted from HRV signals to achieve the highest accuracy of $85.5 \%$ with quadratic discriminant analysis (QDA). Rajput et al. [17] proposed a scheme to identify the low and high risk of hypertension. The scheme yielded classification accuracy $100 \%$ using optimal orthogonal wavelet filter back (OWFB), log, and fractal dimension features extracted from ECG. Despite promising results, this work suffers from a data imbalance problem. The authors in [18] proposed a method to detect ECG hypertensive signals using empirical mode decomposition (EMD) for preprocessing of the signals, yielding an accuracy of $97.7 \%$ through the KNN classifier. The extracted features were selected physically, making this process laborious. The method was only trained on a small data set. A system to detect hypertension using morphological descriptors derived from PPG with $92.31 \%$ accuracy is discussed in [19]. Identification of hypertension patients from ballistocardiograms (BCG) is presented in [20]. The system achieved a mean accuracy of $84.4 \%$ using class association rules (CAR) classifier and morphological features. The BCG signals were collected from patients lying on a smart mattress which has a limited availability.

Medical devices in hospitals can easily get affected by electromagnetic interference (EMI) in a complex electromagnetic environment [21,22]. ECG signals are usually affected by the EMI and preconditioning circuits. Changes in temperature and ambient lighting conditions impact the PPG signal acquisition. PPG signal acquisition is exposed to motion artifacts as well [23]. The frequency of the PPG signal is about 1-3 Hz [24], so it also requires a high order filter for signal denoising. The motivation behind this research was to investigate the feasibility of a new signal modality, i.e., pulse plethysmograph (PuPG). In contrast to PPG that uses light to detect the volume of blood flow in the finger, the PuPG senses the pressure changes in blood flow.

\subsection{Main Contributions}

In this research, novel PuPG signals were used to design the hypertension detection system. The PuPG signal includes considerable cardiac health characteristic information [25-27]. The PuPG signals are recently being used for emotions classification [28] and biometric systems [29] as well. The main contributions of this work are listed as follows:

- This is the first study that used PuPG-based signals for the detection of hypertension.

- To accurately detect the hypertension pattern, we extract a large number of multidomain features from preprocessed PuPG signals through discrete wavelet transform (DWT) and EMD.

- To reduce the feature dimensions and redundancy while improving the discriminative power of features, we proposed a hybrid feature selection and reduction (HFSR) scheme.

- $\quad$ The proposed expert hypertension detection system (EHDS) comprises preprocessing through EMD, followed by the feature extraction, kernel principal component analysis (KPCA), and weighted $k$-nearest neighbor (KNN-W) classifier, achieved an accuracy of $99.4 \%$, sensitivity of $99.6 \%$, and specificity of $99.4 \%$.

The rest of this paper is structured as follows: Section 2 gives details about the materials used in this study. Section 3 describes the details about the methods. Next, we present the results in Sections 4 and 5 discuss the proposed method and its comparative analysis. Section 6 concludes this research paper.

\section{Materials}

\subsection{Data Acquisition}

In this study, a portable pulse plethysmograph (PuPG) sensor PTN-104 (NISensors, iWorx Systems Inc., Dover, UK) in combination with NI myDAQ (National Instruments Corporation, Austin, TX, USA) was used for PuPG data acquisition. PTN-104 sensor is attached to the index finger of the subject to convert pulse pressure into an electrical 
voltage signal. The real-time integral of its output signal produces the same volume pulse signal as the expensive infrared PPG sensor. The PTN-104 is a rugged non-magnetic accelerometer, which is made up of piezoelectric material. IX-myDAQ (National Instruments Corporation, Austin, TX, USA) is a breakout board used for connecting the PTN-104 sensor and myDAQ (National Instruments Corporation, Austin, TX, USA) for data acquisition via mini DIN7 port. NI myDAQ is a low-cost data acquisition tool that converts analog signals to digital format and allows the users to analyze real-time data in NI LabVIEW software (National Instruments Corporation, Austin, TX, USA) on PC. The sampling frequency was set to be $1 \mathrm{kHz}$ for PuPG data acquisition.

It is very essential to highlight the difference between PPG and PuPG signals and sensors. Both of them operate on completely different principles with different input parameters. Table 2 presents a comparison between various properties of both sensors such as input parameters, working principles, and the impact of noise on a signal acquisition. Figure 1 illustrates the output signals acquired from both sensors. It was observed that the PuPG signal carries more information as compared to the PPG signal since multiple frequencies contribute towards the dicrotic notch for PuPG.

Table 2. Difference between PPG and PuPG data acquisition.

\begin{tabular}{lll}
\hline Type & Photoplethysmograph (PPG) Sensor & Pulse Plethysmograph (PuPG) Sensor \\
\hline Input signal & Optical signal & Pressure changes \\
\hline \multirow{3}{*}{ Phenomenon } & $\begin{array}{l}\text { Blood volumetric changes are detected } \\
\text { by measuring the amount of light } \\
\text { transmitted or reflected by the sensor. }\end{array}$ & $\begin{array}{l}\text { Blood volumetric changes are detected by the } \\
\text { piezoelectric material of the sensor as pressure } \\
\text { changes when the blood volume changes. }\end{array}$ \\
\hline \multirow{3}{*}{ Noise Impact } & $\begin{array}{l}\text { Light signal can be easily impacted by } \\
\text { any external light changes. }\end{array}$ & $\begin{array}{l}\text { Piezoelectric material based sensors are normaly } \\
\text { temperature sensitive. }\end{array}$ \\
& $\begin{array}{l}\text { Dirty hand can distort the light } \\
\text { intensities. }\end{array}$ & $\begin{array}{l}\text { Dirty hands or foreign material on hand or fingers } \\
\text { does not have significant impact. }\end{array}$ \\
\hline
\end{tabular}

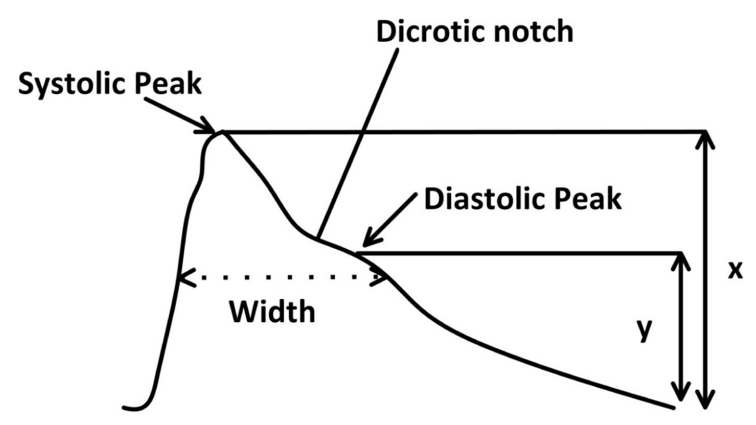

(a) Photoplethysmograph signal

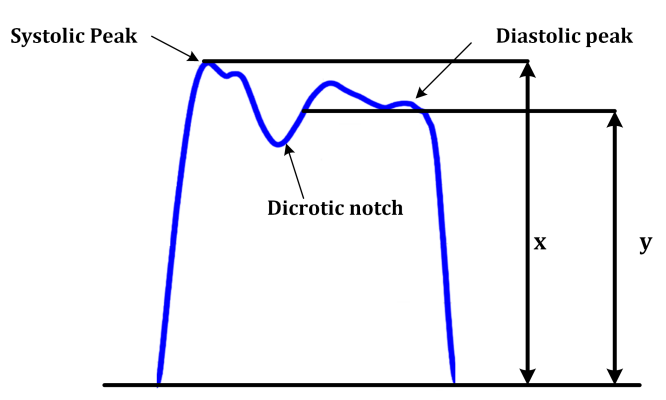

(b) Pulse Plethysmograph signal

Figure 1. Visual comparison of Photoplethysmograph (PPG) and Pulse Plethysmograph (PuPG) signals.

\subsection{Data Set Description}

Raw PuPG signals were acquired from the subjects. The data acquisition was carried out for developing a two-class data bank; one was hypertension and the other normal. A total of 700 signals were collected from hypertension subjects and 709 signals from normal subjects, with a timestamp of $10 \mathrm{~s}$ per signal and a sampling frequency of $1000 \mathrm{~Hz}$. Subjects were advised to keep calm and remain static during data acquisition activity. Informed consent was obtained from all participants included in the research. Recording activity was performed between breakfast and lunch time. None of the involved subjects were smokers or diabetic. Table 3 shows the details of the subjects and the acquired data for this study. Figure 2 shows a comparison of raw PuPG signals collected from a normal subject and a subject suffering from hypertension. Sometimes acquired signals 
(both normal and hypertension) were affected by the circuit noise. The noise/power line distortion incurred due the embedded electronics of data acquisition setup can be seen as a sinusoidal oscillatory component $(50 \mathrm{~Hz})$ in the normal PuPG Hat of Figure 2.

Table 3. Summary of the self-collected PuPG data set.

\begin{tabular}{cccc}
\hline Data Class & Subjects & Age Group & Samples \\
\hline \multirow{2}{*}{ Hypertension } & Male: 29 & Male: $40-76$ & 700 \\
& Female: 27 & Female: $39-59$ & \\
\hline \multirow{2}{*}{ Normal } & Male: 35 & Male: $21-63$ & 709 \\
& Female: 30 & Female: $20-59$ & \\
\hline Overall & 121 & $20-76$ & 1409 \\
\hline
\end{tabular}
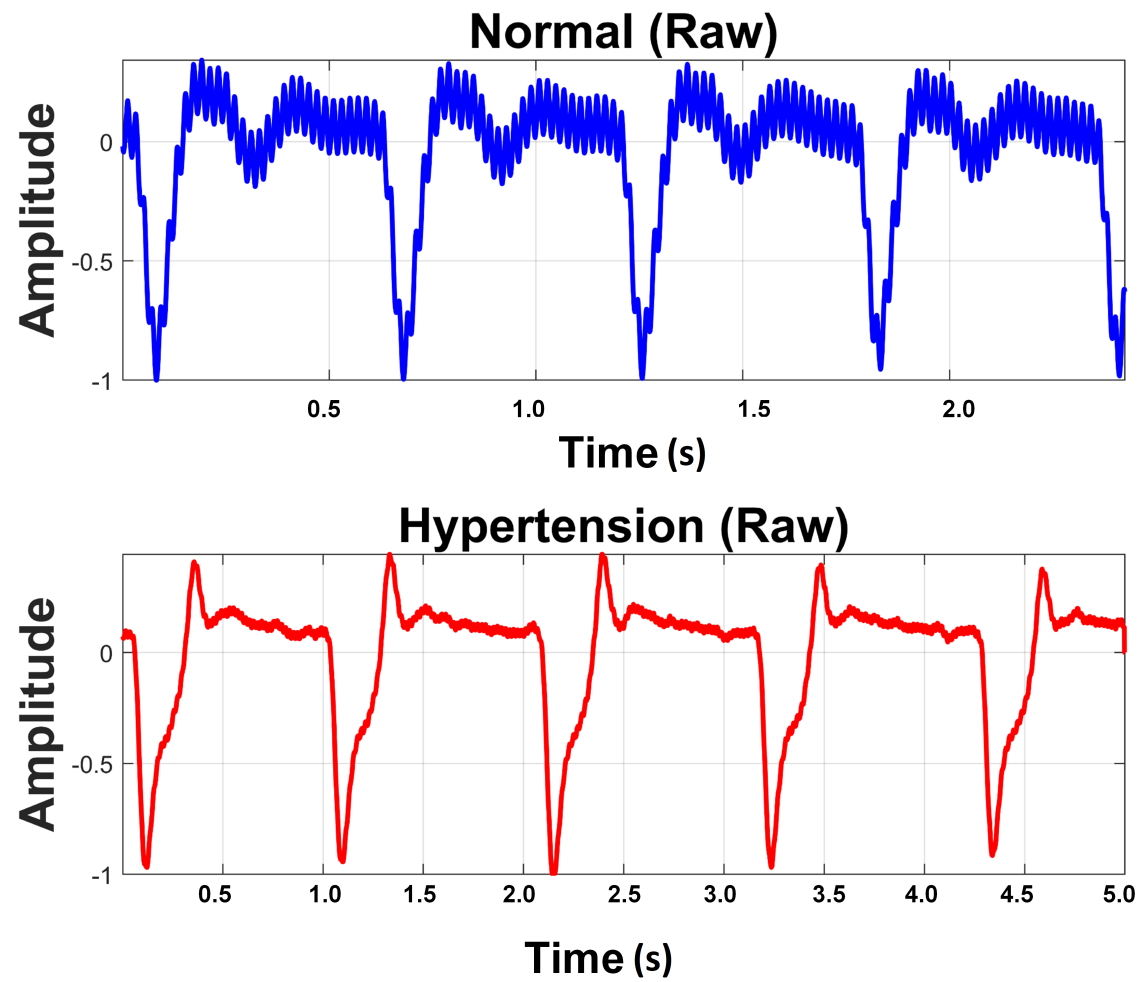

Figure 2. Raw PuPG signals of Normal and Hypertension classes.

\section{Methods}

\subsection{Design of the Study}

The proposed methodology in this research adopts the machine learning paradigm shown in Figure 3. It consists of four main stages, namely (i) preprocessing; (ii) feature extraction; (iii) hybrid feature selection and reduction, and (iv) classification. These stages are separated through a dotted line in Figure 3. Each step is elaborated in detail in forthcoming sections. This research adopts a comparative approach between two pattern analysis frameworks, i.e., method I and method II. Method I is comprised of discrete wavelet transform (DWT)-based preprocessing while method II adopts empirical mode decomposition (EMD) for signal denoising. The rest of the framework for both methods is the same. The feature values extracted, reduced feature vectors, and the performance of the classifiers vary for both methods due to the difference in preprocessing methods. All experiments were performed on MATLAB 2018a (The MathWorks, Inc., Natick, MA, USA) running on a personal computer with Core i7 (Intel Corporation, Santa Clara, CA, USA) processors and 32 GB RAM. 


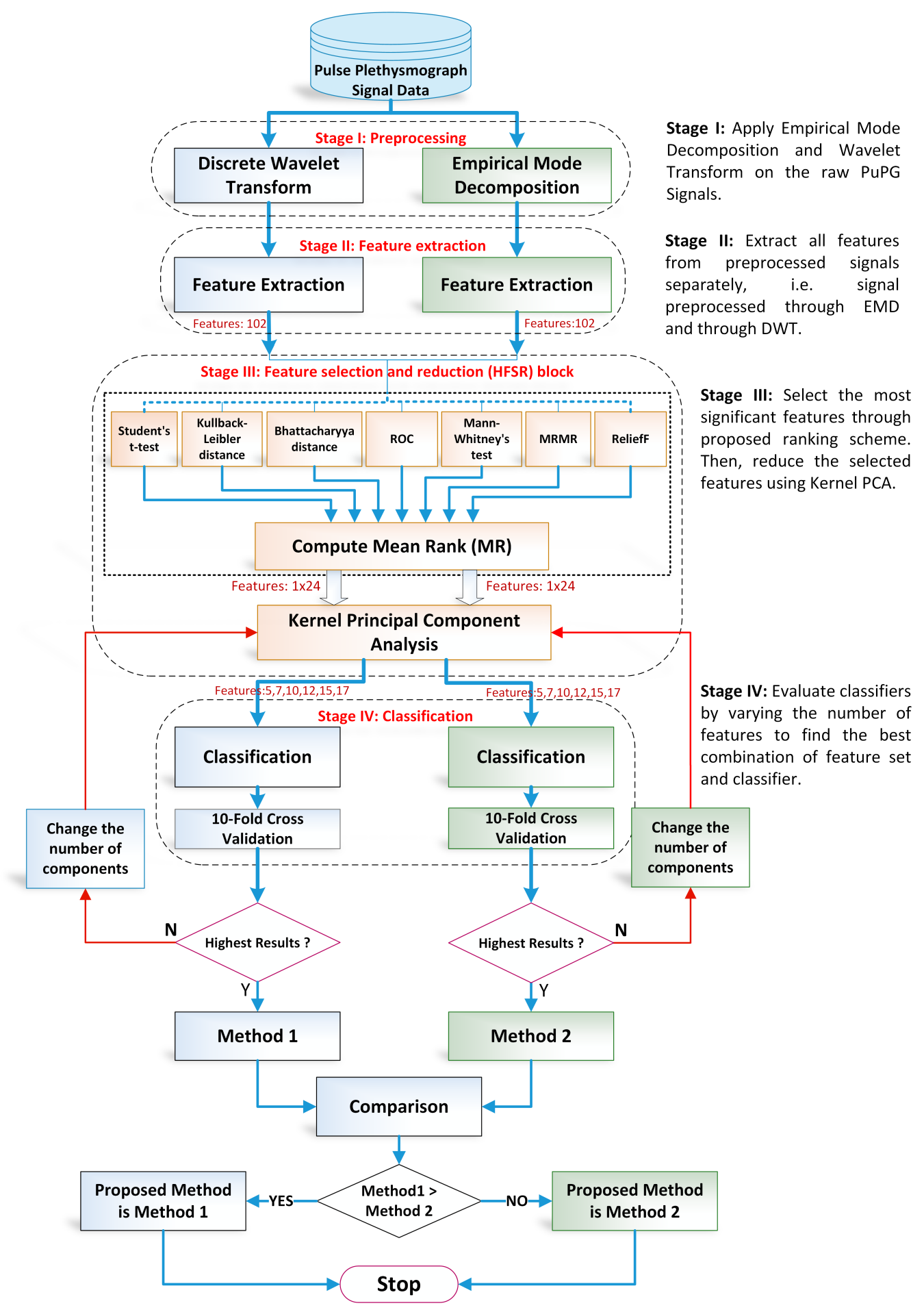

Figure 3. Overall flow chart of the proposed design methodology for detection of hypertension through pulse plethysmograph signals.

1. Preprocessing: It removes the irrelevant information and artifacts from the acquired PuPG signal data of normal and hypertension classes. Method I employs discrete wavelet transform (DWT) for signal denoising through frequency and mean relative energy-based criteria. Method II adopts empirical mode decomposition (EMD) for noise elimination through analysis of mean frequencies and energies of individual signal components extracted from normal and hypertension classes.

2. Feature extraction: It extracts a combination of 102 features from preprocessed PuPG through DWT and EMD separately. These include time, frequency, spectral, texture, 
and cepstral features. The difference between signal classes is best captured through the extraction of a wide range of informative features.

3. Feature selection and reduction: This step eliminates features with redundant information through a hybrid feature selection and reduction (HFSR) method that is a combination of multiple feature ranking and transformation schemes. A high-dimensional feature vector is reduced through a new strategy of the averaging outcome of seven feature ranking methods, thus providing more reliable results. Next, we employed kernel principal component analysis (KPCA) to further decrease the feature dimension and represent significant information in fewer parameters. Extracted features in both method I and II are fed to the HFSR scheme to reduce the dimension of the resultant feature vector.

4. Classification:The final feature vectors extracted in both methods I and II of hypertension and normal classes are fed to a range of different classifiers, i.e, support vector machines (SVM), $k$-nearest neighbors (KNN), ensemble methods, decision trees (DT), and logistic regression (LR). Classification performance of both methods is evaluated through a baseline tenfold cross-validation strategy and compared with 5-, 15-, 20-, and 25-fold cross-validation.

\subsection{Preprocessing}

The acquired PuPG data were contaminated with noise and artifacts and include redundant information (Figure 2). These noise components needs to be eliminated for a robust performance of the proposed system. Therefore, we employed DWT and EMD-based preprocessing for signal denoising. Later on, we compared the preprocessing performance of both methods.

\subsubsection{Discrete Wavelet Transform}

The discrete wavelet transform (DWT) is a widely applied approach in biomedical signal processing applications [30-32]. DWT decomposes a signal into different resolutions by using a combination of high-pass and low-pass filters. Figure 4 illustrates the complete process of wavelet-based denoising [33] adopted in this research. Numerous filter coefficients have been developed for diverse types of signal analysis applications-for instance, Daubechies, Symlets, and Coiflets coefficients, etc.

In this study, we employed the Symlet wavelet due to its similarity with the shape of the PuPG signal under consideration [34,35]. Symlet wavelet yields the best results as compared to others due to its resemblances with the morphological characteristics of the PuPG signal.

Table 4 exhibits information about decomposition levels, frequency ranges, and mean relative energies of normal and hypertension data classes of PuPG signals. It can be observed that $D_{1}, D_{2}, D_{3}$, and $D_{4}$ signal components have high frequency range and include low mean relative energies; therefore, these components were eliminated while reconstructing a denoised signal. This is also endorsed by the fact that the PuPG signal has a very low frequency (normally less than $60 \mathrm{~Hz}$ ). Figure 5 provides a graphical illustration of wavelet decomposition for normal and hypertension PuPG signals. Figure 6 presents the denoised signal generated as a result of applying DWT. High frequency noise visible in raw PuPG signal (Figure 2) is eliminated in the denoised version. 


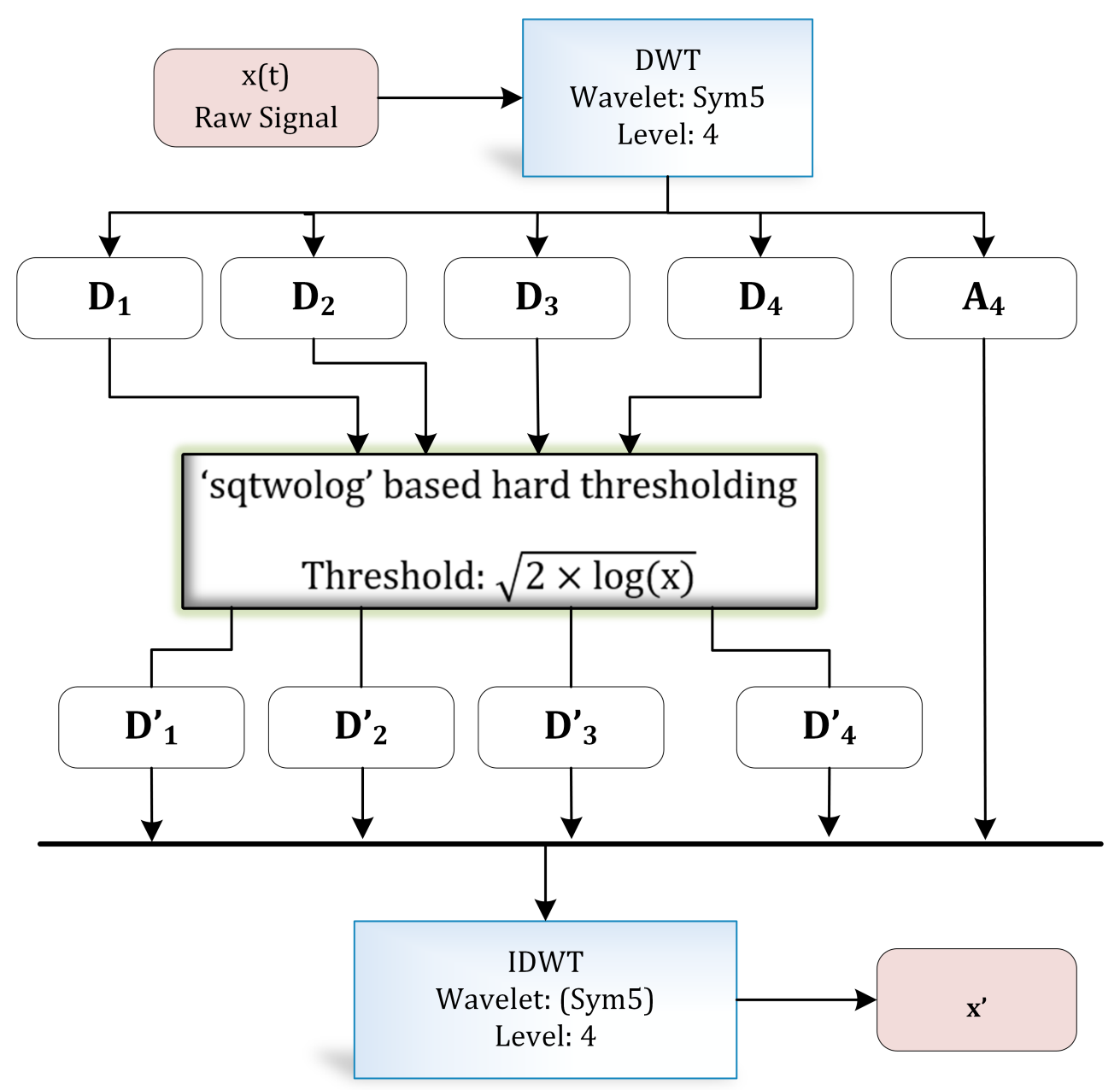

Figure 4. Wavelet-based denoising.

Table 4. Comparison of mean relative energies and frequency ranges of various decomposition levels for Normal and Hypertension classes.

\begin{tabular}{cccc}
\hline \multirow{2}{*}{ Decomposition Levels } & Frequency Range (Hz) & \multicolumn{2}{c}{ Mean Relative Energy (\%) } \\
\cline { 3 - 4 } & & Normal & Hypertension \\
\hline$D_{1}$ & $250-500$ & $0.07 \%$ & $0.59 \%$ \\
$D_{2}$ & $122-256$ & $0.09 \%$ & $0.32 \%$ \\
$D_{3}$ & $61.1-128$ & $0.19 \%$ & $0.35 \%$ \\
$D_{4}$ & $30.6-63.9$ & $0.46 \%$ & $0.49 \%$ \\
$D_{5}$ & $15.3-31.9$ & $1.93 \%$ & $3.10 \%$ \\
$D_{6}$ & $7.65-16$ & $14.77 \%$ & $13.31 \%$ \\
$D_{7}$ & $3.84-7.97$ & $31.03 \%$ & $21.49 \%$ \\
$D_{8}$ & $1.94-3.99$ & $29.11 \%$ & $19.05 \%$ \\
$D_{9}$ & $1.03-1.99$ & $21.11 \%$ & $26.77 \%$ \\
$D_{10}$ & $0.594-0.958$ & $0.16 \%$ & $7.65 \%$ \\
$A_{10}$ & $0-0.431$ & $1.08 \%$ & $6.88 \%$ \\
$A_{4}$ & $0-31.2$ & $99.19 \%$ & $98.26 \%$ \\
\hline
\end{tabular}




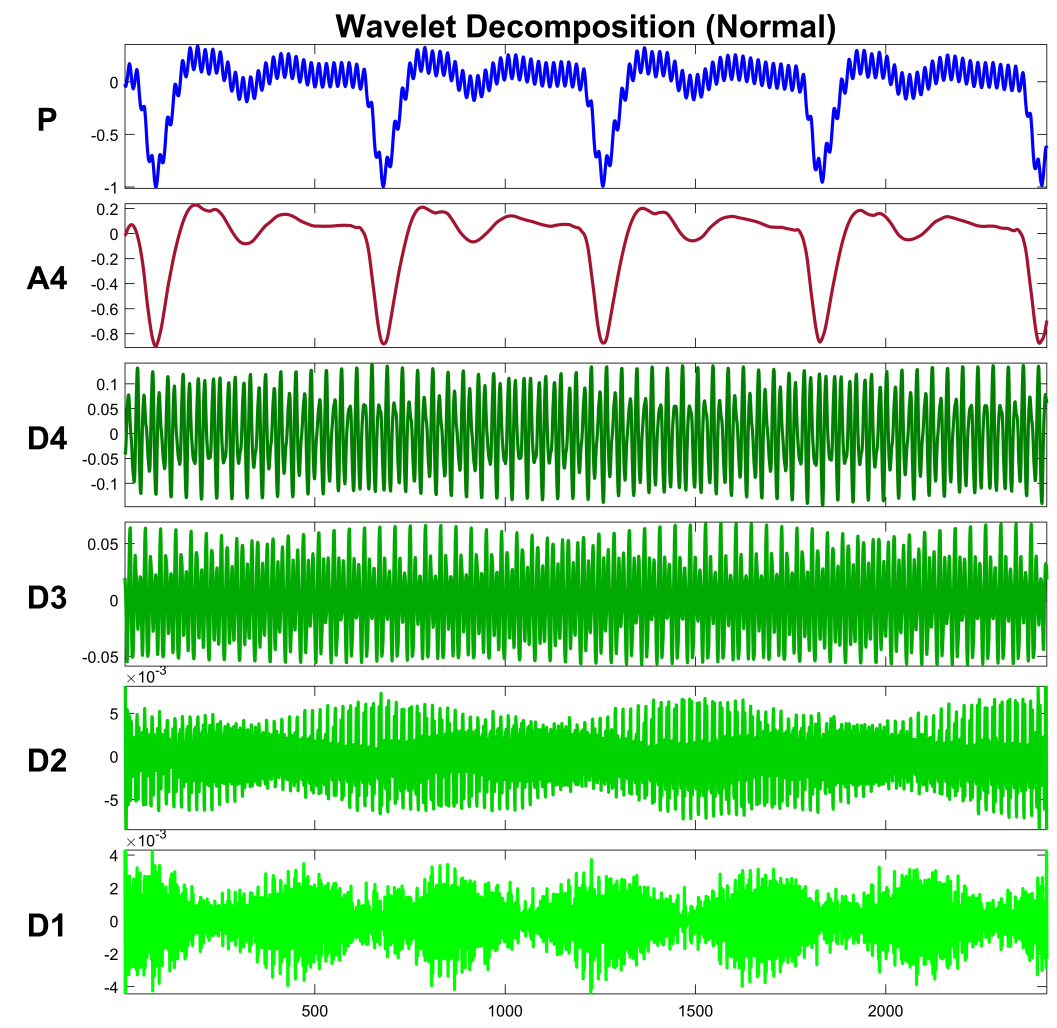

(a) Wavelet decomposition of Normal
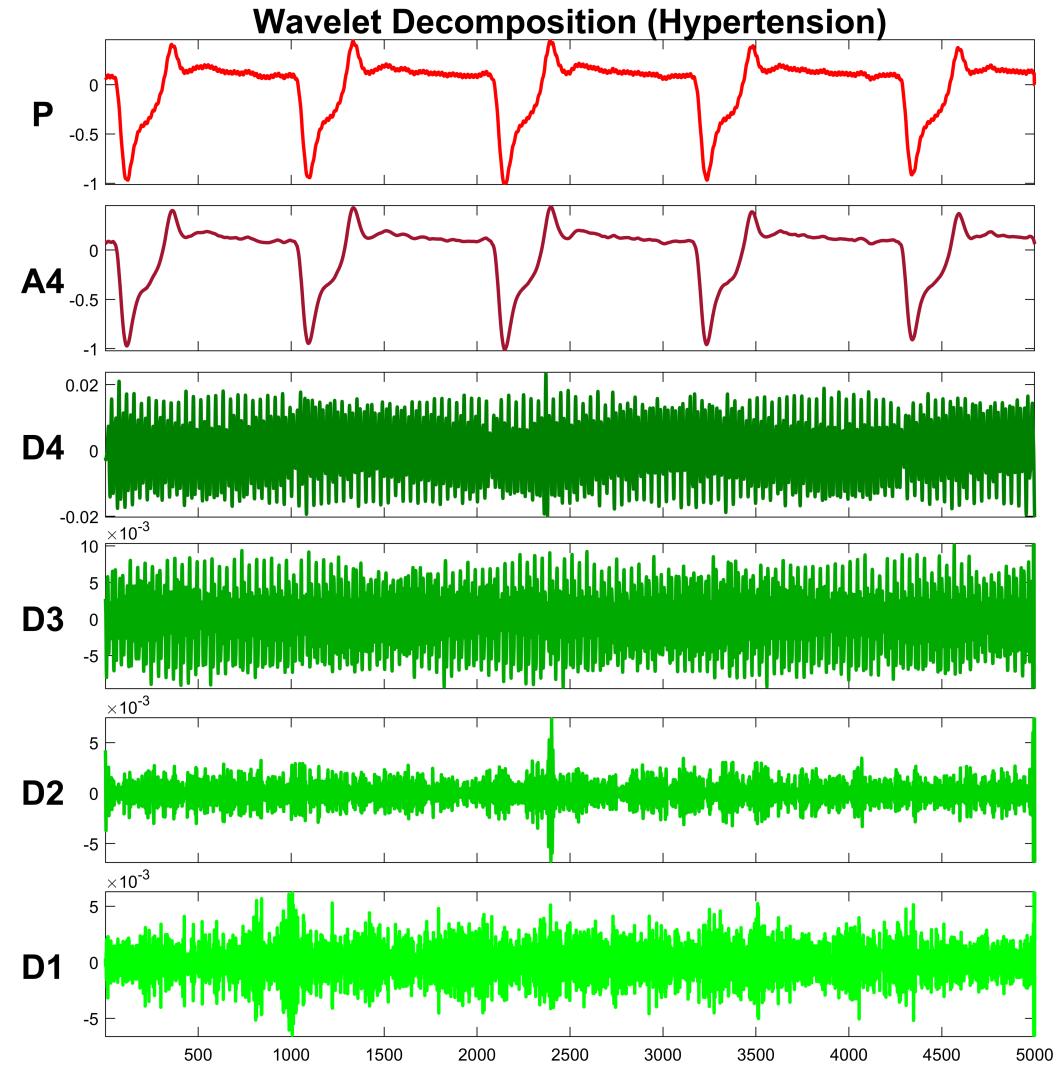

(b) Wavelet decomposition of Hypertension

Figure 5. Wavelet decomposition of raw PuPG signals. 


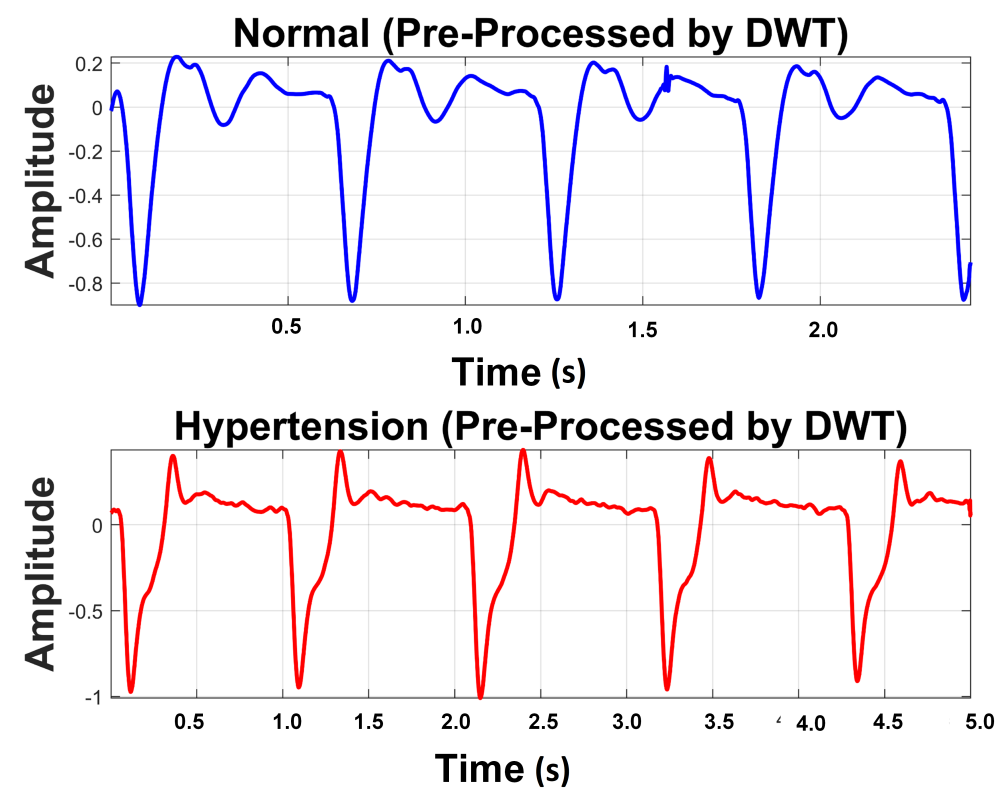

Figure 6. Denoised version of PuPG signal for Normal and Hypertension through DWT.

\subsubsection{Empirical Mode Decomposition}

EMD is an adaptive method that derives fundamental functions directly from the data [36]. EMD does not require any previously known value of the signal for its computation. The principal task for computing EMD of a given signal is to empirically determine the intrinsic oscillatory components through their particular time scales in a signal and subsequently disintegrate the signal into intrinsic mode functions (IMFs) [37]. Therefore, EMD provides remarkably better results for nonlinear and non-stationary biomedical signals.

Selection criteria of IMF have to satisfy two conditions;

- In the entire signal, the total number of local extrema and zero crossings must be equal to each other or differ by a maximum one.

- The average of the envelopes computed through local minima and local maxima must be zero.

The systematic approach to disintegrate the signal into its IMFs is known as the "sifting" process, explained in Figure 7.

The basic objective of applying EMD for preprocessing the PuPG signal was to decompose the distorted signal into its constituent IMFs as depicted in Figure 8. Considering the fact that some IMFs carry discriminative and characteristic information about various data classes while others include redundant and noisy content, the determination of the proper number of IMFs is a crucial step towards creating an effective signal denoising strategy.

It is perceived from Figure 8 and Table 5 that the first IMF includes mainly highfrequency content. Table 5 provides mean frequency and energy information of each IMF for normal and hypertension data classes of the PuPG signal. The first IMF also holds very little mean relative energy components for both classes, i.e., $0.00 \%$ and $1.02 \%$ for normal and hypertension classes, respectively. Therefore, it was discarded while reconstructing the denoised signal. All other IMFs and residual signals were added to form a denoised version of the PuPG signal. Figure 9 illustrates the PuPG signal denoised through the EMD process for normal and hypertension data. It is clear that high frequency noise that was visible in raw PuPG signal (Figure 2) is eliminated now. 


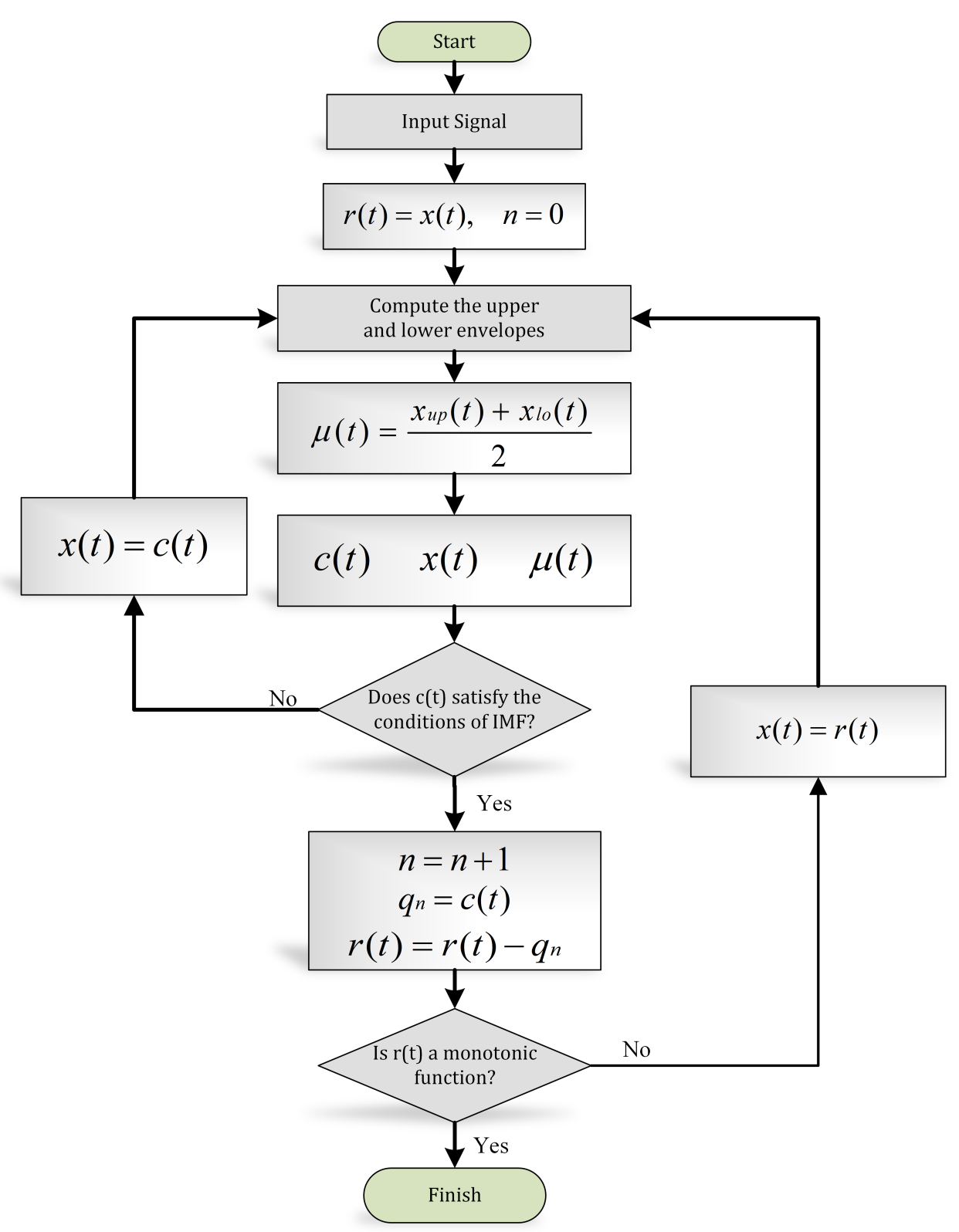

Figure 7. EMD algorithm (flow chart).

Table 5. Comparison of mean relative energies and frequency ranges of various intrinsic mode functions (EMD) for Normal and Hypertension classes. Bold font indicates the selected components.

\begin{tabular}{ccccc}
\hline \multirow{2}{*}{ Components } & \multicolumn{2}{c}{ Normal } & \multicolumn{2}{c}{ Hypertension } \\
\cline { 2 - 5 } & Mean Frequency Range (Hz) & Mean Relative Energy (\%) & Mean Frequency Range (Hz) & Mean Relative Energy (\%) \\
\hline$I M F_{1}$ & $103-483$ & 0.00 & $86.5-484$ & 1.02 \\
$I M F_{2}$ & $\mathbf{1 1 . 3 - 6 0 . 2}$ & $\mathbf{0 . 1 4}$ & $\mathbf{4 0 . 7 - 2 1 9}$ & $\mathbf{0 . 3 5}$ \\
$I M F_{3}$ & $3.09-\mathbf{1 4}$ & 30.34 & $3.3-61$ & 2.04 \\
$I M F_{4}$ & $\mathbf{2 . 9 9 - 1 2 . 2}$ & 4.97 & $3.34-23.3$ & $\mathbf{6 . 6 5}$ \\
$I M F_{5}$ & $\mathbf{1 . 2 8 - 1 0}$ & $\mathbf{2 2 . 7 6}$ & $\mathbf{2 . 9 8 - 1 1 . 4}$ & $\mathbf{1 4 . 9 5}$ \\
Residual & $\mathbf{0 . 1 2 9 - 5 . 5 5}$ & $\mathbf{4 1 . 7 8}$ & $\mathbf{0 . 0 1 9 7 - 4 . 3 3}$ & $\mathbf{7 4 . 9 9}$ \\
\hline
\end{tabular}




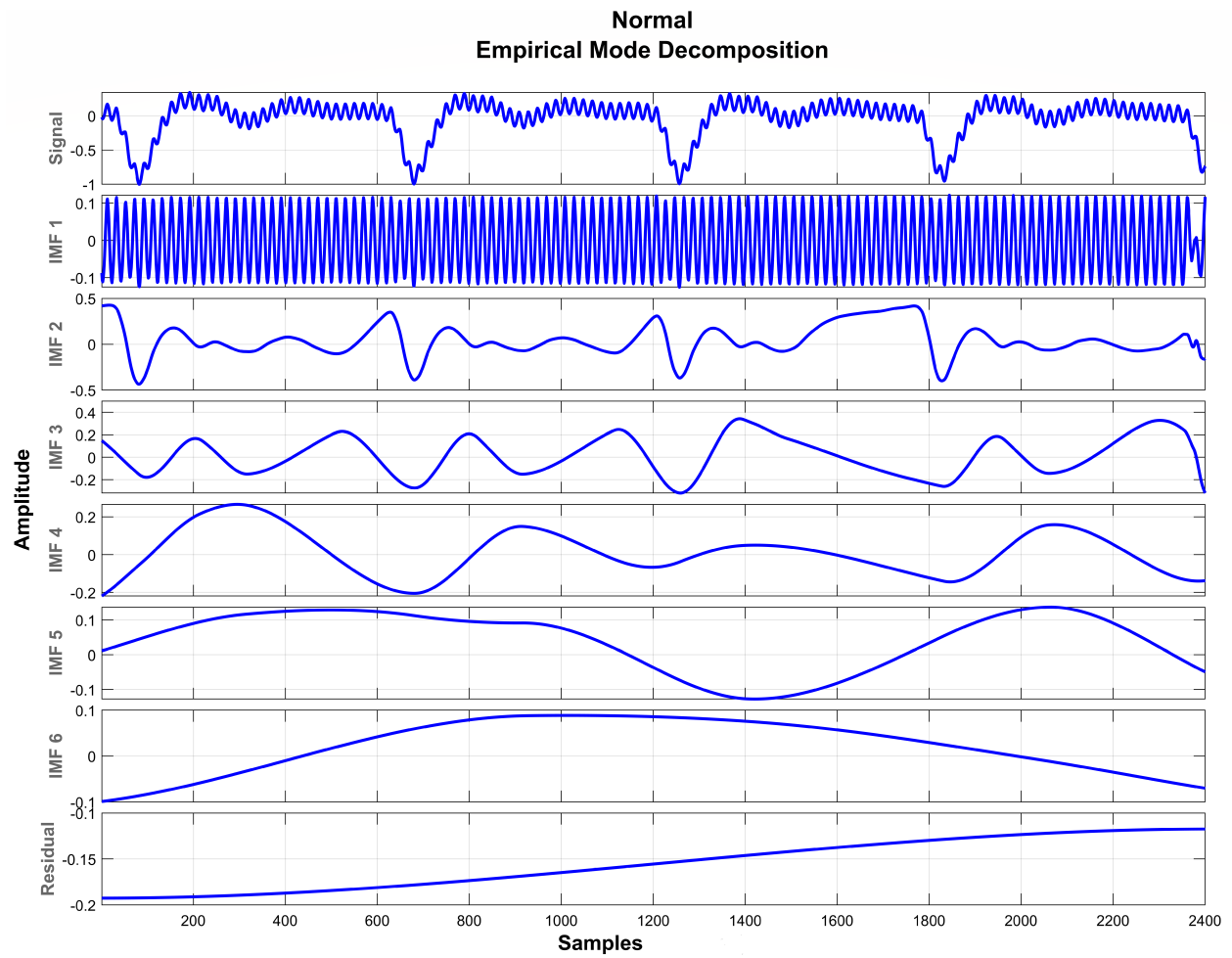

(a) Normal

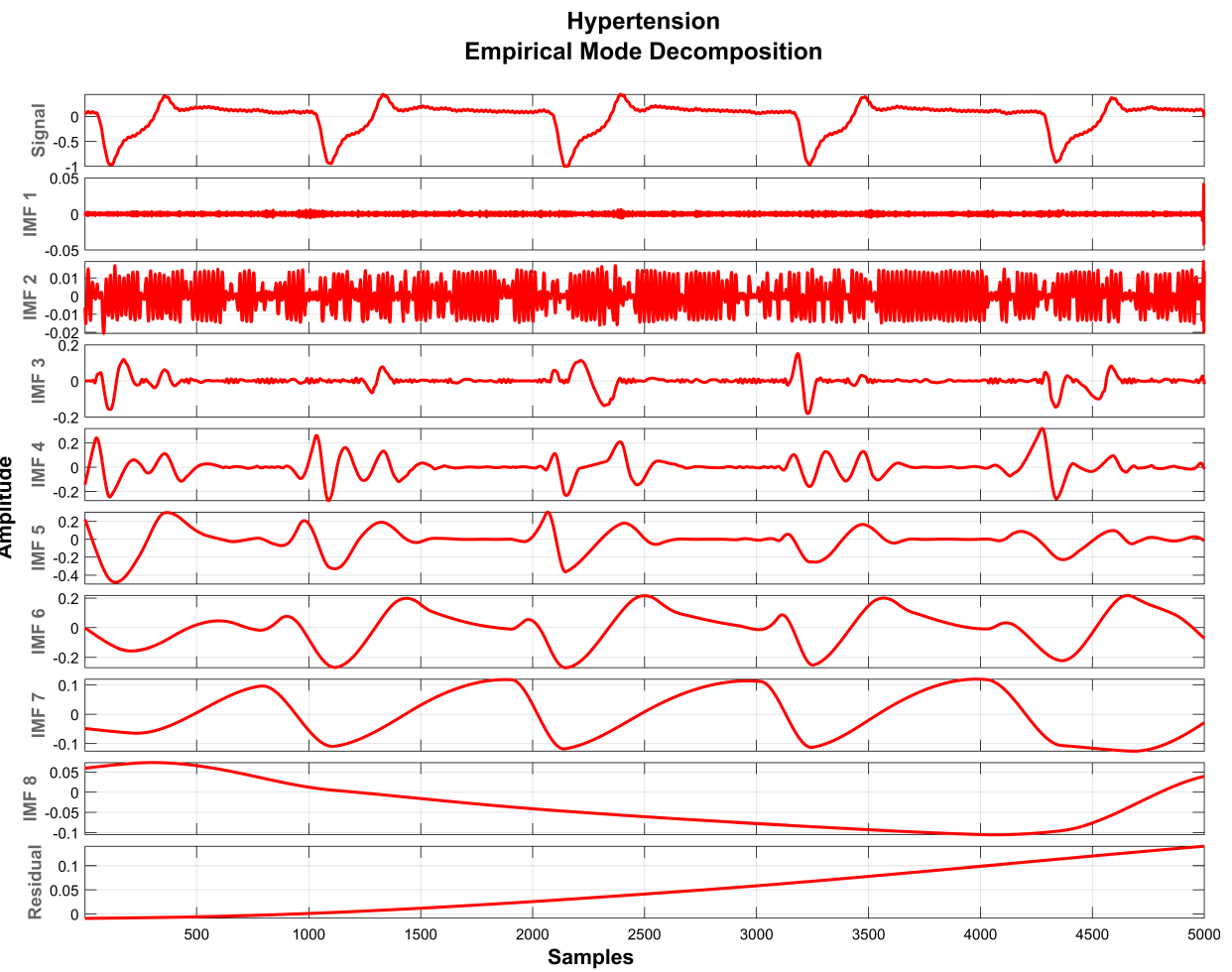

(b) Hypertension

Figure 8. EMD decomposition of raw PuPG signals. 

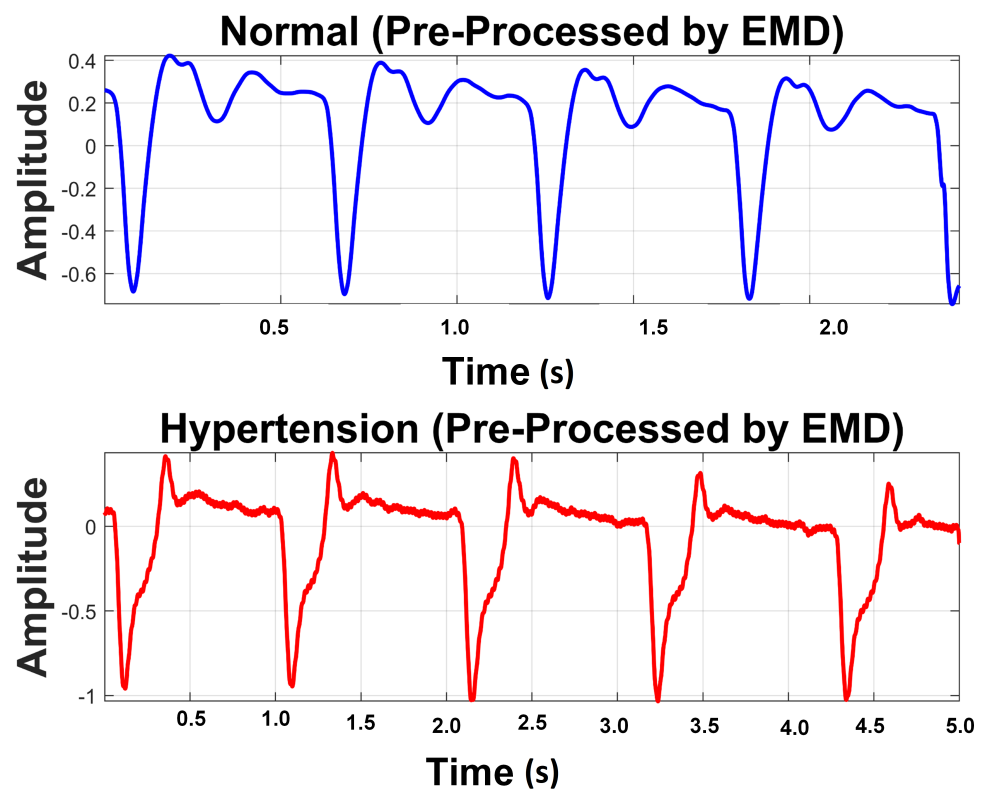

Figure 9. Preprocessed signal using EMD.

\subsection{Feature Extraction}

The objective of the feature extraction stage is to extract significant features from the biomedical signals of various classes that contributes towards an effective classification performance. In this study, a total of 102 features were separately computed from the PuPG signal denoised through DWT and EMD. Table 6 lists all the extracted features along with their statistical measures of mean and standard deviation (STD) for method I (DWT) and method II (EMD). We extracted time domain [38-45], spectral [46,47], fractal and chaos [48,49], chroma [50,51], cepstral [52], and texture features [53] and analyzed them statistically.

Table 6. Statistical data of all extracted features for both methods.

\begin{tabular}{ccccccccc}
\hline & \multicolumn{4}{c}{ Method I } & \multicolumn{3}{c}{ Method II } \\
\hline \multirow{2}{*}{ Feature } & \multicolumn{2}{c}{ Normal } & \multicolumn{2}{c}{ Hypertension } & \multicolumn{2}{c}{ Normal } & \multicolumn{2}{c}{ Hypertension } \\
\cline { 2 - 8 } & Mean & STD & Mean & STD & Mean & STD & Mean & STD \\
\hline Mean & 0.008 & 0.027 & 0.017 & 0.031 & 0.001 & 0.049 & 0.013 & 0.053 \\
Standard Deviation & 0.253 & 0.032 & 0.250 & 0.046 & 0.254 & 0.032 & 0.248 & 0.045 \\
Skewness & -1.959 & 0.522 & -2.144 & 0.651 & -1.997 & 0.576 & -2.220 & 0.641 \\
Kurtosis & 6.993 & 1.499 & 8.083 & 3.864 & 7.139 & 1.688 & 8.297 & 3.944 \\
Peak to Peak Value & 1.380 & 0.199 & 1.398 & 0.140 & 1.377 & 0.178 & 1.375 & 0.144 \\
Root Mean Square & 0.255 & 0.033 & 0.252 & 0.047 & 0.258 & 0.038 & 0.254 & 0.046 \\
Crest Factor & 1.659 & 0.939 & 1.635 & 0.381 & 1.587 & 0.991 & 1.523 & 0.536 \\
Shape Factor & 1.484 & 0.134 & 1.458 & 0.159 & 1.522 & 0.163 & 1.465 & 0.167 \\
Impulse Factor & 2.435 & 1.297 & 2.382 & 0.593 & 2.371 & 1.376 & 2.171 & 0.632 \\
Margin Factor & 15.22 & 14.64 & 15.12 & 7.27 & 15.16 & 15.56 & 13.39 & 6.45 \\
Energy & 389.6 & 209.7 & 437.4 & 207.6 & 393.9 & 207.4 & 448.5 & 222.5 \\
Peak to RMS Value & 3.894 & 0.623 & 4.094 & 0.991 & 3.921 & 0.663 & 4.108 & 1.102 \\
Root Sum of Squares & 18.933 & 5.600 & 20.292 & 5.089 & 19.069 & 5.525 & 20.480 & 5.412 \\
Shannon Energy & 549.7 & 312.3 & 618.2 & 279.7 & 526.6 & 295.8 & 686.5 & 414.4 \\
Log Energy & $-27,888$ & 15,515 & $-34,569$ & 18,644 & $-28,509$ & 16,289 & $-32,613$ & 16,387 \\
Mean Absolute Deviation & 0.169 & 0.026 & 0.169 & 0.043 & 0.169 & 0.028 & 0.168 & 0.043 \\
Median Absolute Deviation & 0.074 & 0.026 & 0.071 & 0.030 & 0.071 & 0.033 & 0.059 & 0.024 \\
\hline
\end{tabular}


Table 6. Cont.

\begin{tabular}{|c|c|c|c|c|c|c|c|c|}
\hline \multirow{3}{*}{ Feature } & \multicolumn{4}{|c|}{ Method I } & \multicolumn{4}{|c|}{ Method II } \\
\hline & \multicolumn{2}{|c|}{ Normal } & \multicolumn{2}{|c|}{ Hypertension } & \multicolumn{2}{|c|}{ Normal } & \multicolumn{2}{|c|}{ Hypertension } \\
\hline & Mean & STD & Mean & STD & Mean & STD & Mean & STD \\
\hline Average Frequency & 0.002 & 0.001 & 0.001 & 0.001 & 0.002 & 0.003 & 0.002 & 0.002 \\
\hline Jitter & 137.1 & 180.9 & 85.5 & 150.4 & 159.0 & 248.8 & 52.0 & 95.8 \\
\hline Spectral Mean & 3.144 & 5.928 & 0.771 & 1.960 & 8.623 & 15.700 & 2.764 & 6.372 \\
\hline Spectral Standard Deviation & 3.401 & 4.833 & 1.549 & 3.463 & 7.571 & 11.498 & 3.031 & 6.016 \\
\hline Spectral Skewness & 2.361 & 1.496 & 3.763 & 1.295 & 0.996 & 1.360 & 1.797 & 1.908 \\
\hline Specral Kurtosis & 11.331 & 8.235 & 20.190 & 9.589 & 5.588 & 4.947 & 10.564 & 8.751 \\
\hline Spectral Centroid & 9.442 & 0.202 & 9.915 & 1.481 & 9.771 & 1.576 & 10.768 & 2.216 \\
\hline Spectral Flux & 0.008 & 0.002 & 0.008 & 0.002 & 0.008 & 0.002 & 0.008 & 0.002 \\
\hline Spectral Roll-off & 91.699 & 1.010 & 96.587 & 8.383 & 97.036 & 10.371 & 136.257 & 44.372 \\
\hline Spectral Flatness & 0.025 & 0.014 & 0.051 & 0.035 & 0.063 & 0.065 & 0.171 & 0.138 \\
\hline Spectral Crest & 0.642 & 0.012 & 0.621 & 0.040 & 0.631 & 0.030 & 0.593 & 0.059 \\
\hline Spectral Decrease & -4.333 & 0.228 & -4.013 & 0.658 & -4.169 & 0.538 & -3.656 & 0.830 \\
\hline Spectral Slope & -0.023 & 0.003 & -0.023 & 0.003 & -0.024 & 0.003 & -0.023 & 0.005 \\
\hline Spectral Spread & 18.505 & 0.199 & 19.029 & 1.178 & 19.328 & 1.617 & 23.521 & 5.818 \\
\hline Mean Frequency & 4.347 & 0.949 & 4.999 & 3.451 & 4.989 & 3.063 & 6.446 & 4.440 \\
\hline Median Frequency & 3.574 & 0.803 & 2.972 & 0.880 & 3.811 & 3.573 & 2.846 & 0.753 \\
\hline Spurious-free Dynamic Range & 3.073 & 6.019 & 2.196 & 2.107 & 3.163 & 6.451 & 2.056 & 1.812 \\
\hline Signal to Noise Distortion & -0.885 & 6.041 & -2.036 & 3.101 & -0.755 & 6.819 & -2.097 & 3.030 \\
\hline Total Harmonic Distortions & -2.376 & 5.452 & -0.938 & 4.964 & -3.144 & 6.562 & -0.396 & 4.110 \\
\hline 1st Coeffient of MFCC & -44.99 & 0.37 & -44.716 & 0.512 & -44.84 & 0.60 & -44.45 & 0.80 \\
\hline 2nd Coeffient of MFCC & 6.268 & 0.523 & 6.661 & 0.714 & 6.480 & 0.846 & 7.028 & 1.122 \\
\hline 3rd Coeffient of MFCC & 5.976 & 0.499 & 6.350 & 0.683 & 6.169 & 0.802 & 6.690 & 1.066 \\
\hline 4th Coeffient of MFCC & 5.508 & 0.462 & 5.851 & 0.634 & 5.671 & 0.733 & 6.148 & 0.976 \\
\hline 1st Coeffient of GFCC & -7.183 & 0.430 & -6.762 & 0.610 & -7.027 & 0.793 & -6.220 & 1.266 \\
\hline 2nd Coeffient of GFCC & 1.844 & 0.063 & 1.869 & 0.071 & 1.522 & 0.419 & 1.119 & 0.574 \\
\hline 3rd Coeffient of GFCC & 0.553 & 0.138 & 0.367 & 0.269 & 0.643 & 0.105 & 0.492 & 0.206 \\
\hline 4th Coeffient of GFCC & 0.301 & 0.024 & 0.266 & 0.033 & 0.392 & 0.109 & 0.408 & 0.109 \\
\hline 1st Coefficient of Chroma Vector & 0.383 & 0.235 & 0.750 & 0.501 & 0.653 & 0.532 & 2.126 & 1.930 \\
\hline 2nd Coefficient of Chroma Vector & 0.416 & 0.258 & 0.773 & 0.518 & 0.663 & 0.546 & 2.130 & 1.948 \\
\hline 3rd Coefficient of Chroma Vector & 0.433 & 0.269 & 0.842 & 0.575 & 0.742 & 0.672 & 2.129 & 2.144 \\
\hline 4th Coefficient of Chroma Vector & 0.623 & 0.378 & 1.297 & 0.942 & 0.700 & 0.568 & 2.011 & 2.046 \\
\hline 5th Coefficient of Chroma Vector & 0.564 & 0.337 & 1.212 & 0.872 & 0.691 & 0.534 & 2.044 & 1.935 \\
\hline 6th Coefficient of Chroma Vector & 0.527 & 0.320 & 1.230 & 0.987 & 0.748 & 0.563 & 2.227 & 2.267 \\
\hline 7th Coefficient of Chroma Vector & 0.483 & 0.296 & 1.069 & 0.760 & 0.705 & 0.524 & 2.107 & 1.893 \\
\hline 8th Coefficient of Chroma Vector & 0.451 & 0.279 & 0.982 & 0.696 & 0.686 & 0.528 & 2.071 & 1.863 \\
\hline 9th Coefficient of Chroma Vector & 0.429 & 0.268 & 0.908 & 0.638 & 0.679 & 0.529 & 2.099 & 1.929 \\
\hline 10th Coefficient of Chroma Vector & 0.400 & 0.251 & 0.878 & 0.609 & 0.651 & 0.537 & 2.087 & 1.848 \\
\hline 11th Coefficient of Chroma Vector & 0.373 & 0.232 & 0.776 & 0.537 & 0.668 & 0.522 & 2.106 & 1.954 \\
\hline 12th Coefficient of Chroma Vector & 0.348 & 0.225 & 0.705 & 0.474 & 0.622 & 0.522 & 2.078 & 1.890 \\
\hline Enhanced Mean Absolute Value & 0.297 & 0.039 & 0.302 & 0.055 & 0.294 & 0.052 & 0.301 & 0.049 \\
\hline Enhanced Wavelength & 236.4 & 133.5 & 413.7 & 319.8 & 284.3 & 198.6 & 665.7 & 515.0 \\
\hline Wavelength & 36.83 & 21.94 & 85.59 & 96.94 & 54.42 & 46.94 & 193.47 & 186.80 \\
\hline Slope Sign Change & 45.1 & 86.9 & 508.4 & 549.0 & 1039.5 & 1657.6 & 3463.1 & 2792.1 \\
\hline Average Amplitude Change & 0.006 & 0.003 & 0.010 & 0.009 & 0.009 & 0.009 & 0.021 & 0.018 \\
\hline Difference Absolute Std. Dev. & 0.009 & 0.003 & 0.013 & 0.010 & 0.014 & 0.010 & 0.027 & 0.021 \\
\hline Log Detector & 0.108 & 0.030 & 0.118 & 0.037 & 0.107 & 0.043 & 0.117 & 0.036 \\
\hline Modified Mean Absolute Value & 0.130 & 0.025 & 0.133 & 0.034 & 0.130 & 0.033 & 0.132 & 0.030 \\
\hline Modified Mean Absolute Value 2 & 0.083 & 0.022 & 0.089 & 0.026 & 0.084 & 0.027 & 0.087 & 0.020 \\
\hline Pulse Percentage Rate & 0.939 & 0.029 & 0.953 & 0.027 & 0.937 & 0.045 & 0.957 & 0.035 \\
\hline
\end{tabular}


Table 6. Cont.

\begin{tabular}{|c|c|c|c|c|c|c|c|c|}
\hline \multirow{3}{*}{ Feature } & \multicolumn{4}{|c|}{ Method I } & \multicolumn{4}{|c|}{ Method II } \\
\hline & \multicolumn{2}{|c|}{ Normal } & \multicolumn{2}{|c|}{ Hypertension } & \multicolumn{2}{|c|}{ Normal } & \multicolumn{2}{|c|}{ Hypertension } \\
\hline & Mean & STD & Mean & STD & Mean & STD & Mean & STD \\
\hline Simple Square Integral & 389.6 & 209.7 & 437.4 & 207.6 & 393.9 & 207.4 & 448.5 & 222.5 \\
\hline Willison Amplitude & 1153.6 & 765.6 & 2670.8 & 2603.9 & 1836.0 & 1707.6 & 4551.3 & 3502.4 \\
\hline Maximum Fractal Length & -0.463 & 0.384 & -0.136 & 0.740 & -0.174 & 0.594 & 0.428 & 1.121 \\
\hline Root Squared Zero Order Moment & 2.592 & 0.032 & 2.600 & 0.026 & 2.593 & 0.031 & 2.601 & 0.028 \\
\hline Root Squared 2nd Order Moment & 2.068 & 0.066 & 2.036 & 0.062 & 1.984 & 0.115 & 1.913 & 0.130 \\
\hline Root Squared 4th Order Moment & 2.045 & 0.077 & 2.001 & 0.078 & 1.891 & 0.159 & 1.794 & 0.205 \\
\hline Sparseness & 0.535 & 0.064 & 0.582 & 0.086 & 0.655 & 0.137 & 0.747 & 0.188 \\
\hline Irregularity Factor & -0.464 & 0.037 & -0.445 & 0.047 & -0.446 & 0.058 & -0.406 & 0.061 \\
\hline Waveform Length Ratio & -0.065 & 0.703 & -0.354 & 0.230 & -0.648 & 0.604 & -0.721 & 0.727 \\
\hline Complexity & 0.502 & 0.222 & 0.706 & 0.253 & 0.897 & 0.524 & 1.314 & 0.515 \\
\hline Mobility & 0.038 & 0.011 & 0.057 & 0.038 & 0.055 & 0.035 & 0.115 & 0.079 \\
\hline Higuchi's Fractal Dimension & 1.054 & 0.052 & 1.149 & 0.119 & 1.183 & 0.240 & 1.490 & 0.401 \\
\hline Katz Fractal Dimension & 1.000 & 0.000 & 1.000 & 0.000 & 1.000 & 0.000 & 1.000 & 0.000 \\
\hline Lyapunov Exponent & 437.4 & 49.5 & 394.8 & 49.3 & 362.9 & 78.8 & 239.1 & 92.2 \\
\hline Approximate Entropy & 0.104 & 0.049 & 0.155 & 0.134 & 0.127 & 0.080 & 0.316 & 0.312 \\
\hline Correlation Dimension & 1.687 & 0.168 & 1.733 & 0.191 & 1.676 & 0.202 & 1.731 & 0.300 \\
\hline 1st Coefficient of LTP & 259.6 & 128.8 & 291.5 & 122.1 & 259.6 & 128.8 & 291.5 & 122.1 \\
\hline 2nd Coefficient of LTP & 40.790 & 30.943 & 56.809 & 35.998 & 40.790 & 30.943 & 56.809 & 35.998 \\
\hline 3rd Coefficient of LTP & 24.574 & 23.717 & 41.723 & 32.083 & 24.574 & 23.717 & 41.723 & 32.083 \\
\hline 4th Coefficient of LTP & 16.381 & 15.761 & 30.738 & 22.667 & 16.381 & 15.761 & 30.738 & 22.667 \\
\hline 5th Coefficient of LTP & 18.472 & 15.755 & 33.411 & 24.881 & 18.472 & 15.755 & 33.411 & 24.881 \\
\hline 6th Coefficient of LTP & 25.205 & 21.607 & 45.518 & 33.715 & 25.205 & 21.607 & 45.518 & 33.715 \\
\hline 7th Coefficient of LTP & 17.699 & 16.352 & 29.035 & 22.609 & 17.699 & 16.352 & 29.035 & 22.609 \\
\hline 8th Coefficient of LTP & 26.261 & 23.075 & 39.645 & 28.386 & 26.261 & 23.075 & 39.645 & 28.386 \\
\hline 9th Coefficient of LTP & 47.483 & 32.844 & 58.163 & 35.132 & 47.483 & 32.844 & 58.163 & 35.132 \\
\hline 10th Coefficient of LTP & 207.278 & 94.769 & 201.809 & 72.537 & 207.278 & 94.769 & 201.809 & 72.537 \\
\hline 11th Coefficient of LTP & 269.5 & 137.7 & 302.1 & 121.7 & 269.5 & 137.70 & 302.1 & 121.7 \\
\hline 12th Coefficient of LTP & 41.733 & 31.354 & 59.773 & 38.092 & 41.733 & 31.354 & 59.773 & 38.092 \\
\hline 13th Coefficient of LTP & 24.006 & 23.096 & 41.071 & 31.294 & 24.006 & 23.096 & 41.071 & 31.294 \\
\hline 14th Coefficient of LTP & 16.784 & 15.900 & 30.199 & 23.471 & 16.784 & 15.900 & 30.199 & 23.471 \\
\hline 15th Coefficient of LTP & 18.506 & 15.140 & 33.390 & 25.583 & 18.506 & 15.140 & 33.390 & 25.583 \\
\hline 16th Coefficient of LTP & 26.148 & 22.755 & 44.177 & 31.682 & 26.148 & 22.755 & 44.177 & 31.682 \\
\hline 17th Coefficient of LTP & 16.898 & 16.559 & 30.149 & 21.910 & 16.898 & 16.559 & 30.149 & 21.910 \\
\hline 18th Coefficient of LTP & 25.790 & 22.892 & 39.312 & 26.784 & 25.790 & 22.892 & 39.312 & 26.784 \\
\hline 19th Coefficient of LTP & 46.534 & 33.045 & 56.298 & 33.647 & 46.534 & 33.045 & 56.298 & 33.647 \\
\hline 20th Coefficient of LTP & 197.773 & 85.090 & 191.858 & 74.487 & 197.773 & 85.090 & 191.858 & 74.487 \\
\hline
\end{tabular}

These features were subjected to the feature selection step (HFSR) to recognize the features with maximum discriminative content among normal and hypertension classes.

\subsection{Hybrid Feature Selection and Reduction}

Feature selection is one of the key steps in the modern pattern recognition and machine learning paradigms. The extracted features may include redundant information and irrelevant and noisy parameters. A two-stage hybrid feature selection and reduction (HFSR) strategy was designed to select and transform the best distinctive features as shown in Figure 10. The first stage ranks the input features through seven different methods and the second stage transforms the selected ranked features to further reduce dimensionality. 


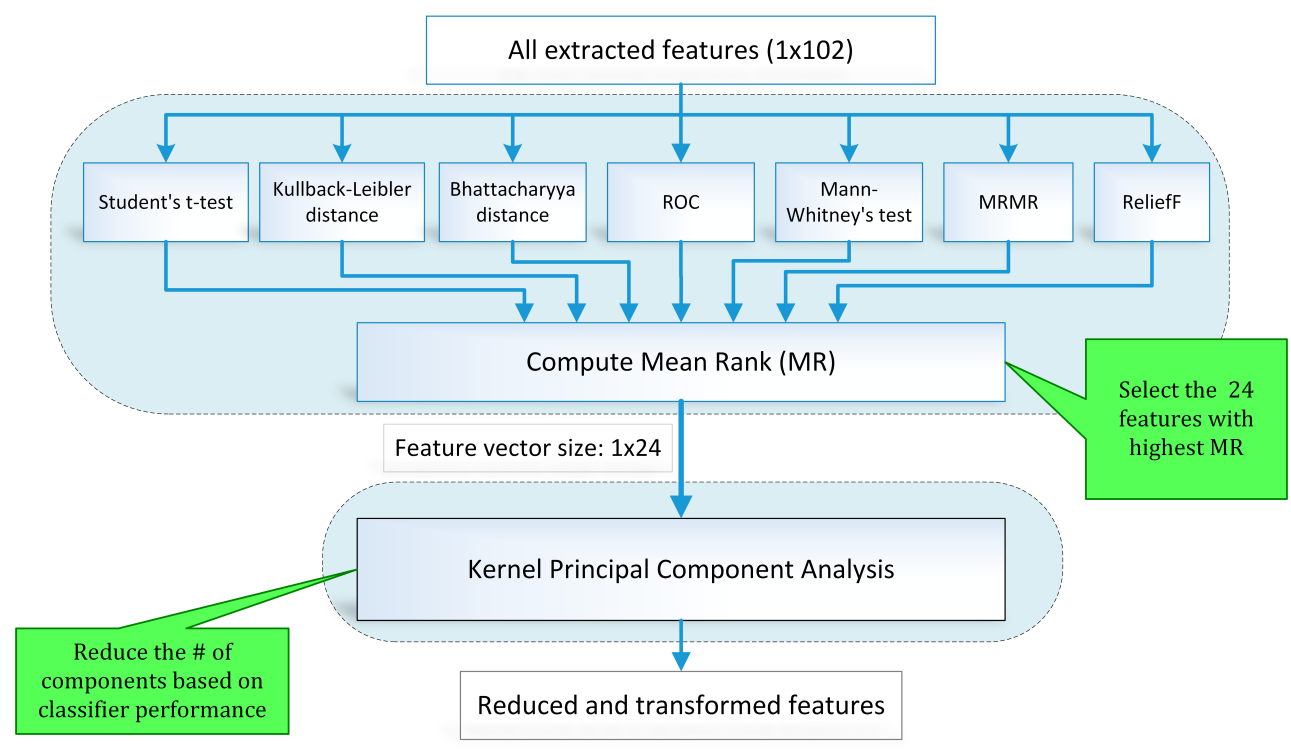

Figure 10. Block diagram (feature selection and reduction method).

\subsubsection{Feature Selection Scheme}

Feature selection routines serve to enhance the performance of classifiers by reducing the feature dimensions as well as decreasing the computational time $[54,55]$. Feature selection methods are categorized as filter methods and wrapper methods. Filter type feature selection methods employ feature ranking techniques based on the applied statistical measure for selecting a suitable feature. In wrapper type feature selection techniques, a feature subset is selected recursively based on the overall model performance. The selection criterion computes the variation in model performance that decides the addition or removal of a feature from the subset.

To address the limitations of individual feature selection approaches, we employed a hybrid scheme of feature selection by combining seven feature ranking methods through a voting strategy. Figure 10 illustrates the hybrid scheme of feature selection and reduction. In this scheme, seven state-of-the-art feature ranking techniques, namely student t-test (TT), Kullback-Leibler distance (KLD) [56], Bhattacharya distance (BD) [57], MannWhitney's test (MWT), ReliefF (RRF) [58], minimum redundancy maximum relevance (MRMR) [59,60], and receiver operating characteristic curve (ROC) were employed to rank the feature individually. Ranking assigned to each feature by all feature ranking methods is combined to calculate the mean rank (MR) value. A threshold is applied to MR value for feature selection.

Table 7 provides the sorted lists of the best forty features with the highest MR values for method I (features extracted from signal preprocessed through DWT). Rank assigned to individual features by each ranking method is also computed. The top 24 features are highlighted in Table 6 were forwarded to the next stage. It was perceived that if a feature ranking method assigns a high rank to a particular feature that failed to get high scores from other methods, it gets rejected due to the hybrid scheme of feature selection. For instance, consider the Root Sum of Squares feature that received the rank value of 99 from the ROC method, but gets scores of 53, 49, 52, 18, 58, and 72 from TT, KLD, BD, MWT, MRMR, and RRF, respectively. It achieved an MR value of 57.29 that is below the selection criterion, so it was rejected from the final feature vector of $1 \times 24$ dimensions. Table 8 enlists the top forty features with the highest MR values for method II, i.e., features extracted from the signal preprocessed through EMD. The rank value assigned by an individual feature ranking method to a specific feature can be examined. One to one comparison of the top ten MR values of method I in Table 7 and method II in Table 8 reveals that the magnitude of MR values of method II (81-70) is higher than that for method I (73-67). 
Table 7. List of features extracted for method I and sorted with respect to mean rank (MR) value. Bold font indicates the top 24 ranked features.

\begin{tabular}{|c|c|c|c|c|c|c|c|c|}
\hline Feature & TT & KLD & BD & ROC & MWT & MRMR & RRF & MR \\
\hline 3rd Coefficient of LTP & 12 & 93 & 93 & 83 & 32 & 102 & 98 & 73.29 \\
\hline 6th Coefficient of Chroma Vector & 38 & 98 & 76 & 79 & 78 & 94 & 49 & 73.14 \\
\hline Lyapunov Exponent & 93 & 92 & 92 & 70 & 81 & 10 & 70 & 72.57 \\
\hline Sparseness & 70 & 90 & 90 & 30 & 62 & 79 & 84 & 72.14 \\
\hline Jitter & 78 & 58 & 58 & 74 & 77 & 85 & 74 & 72.00 \\
\hline 9th Coefficient of LTP & 57 & 83 & 83 & 75 & 82 & 92 & 24 & 70.86 \\
\hline Spectral Decrease & 62 & 28 & 61 & 85 & 75 & 93 & 83 & 69.57 \\
\hline 4th Coefficient of MFCC & 96 & 41 & 41 & 95 & 40 & 72 & 99 & 69.14 \\
\hline Irregularity & 35 & 94 & 100 & 57 & 88 & 90 & 13 & 68.14 \\
\hline 1st Coeffient of MFCC & 99 & 23 & 23 & 100 & 37 & 96 & 97 & 67.86 \\
\hline Waveform Length Ratio & 13 & 100 & 94 & 91 & 9 & 100 & 66 & 67.57 \\
\hline 3rd Coeffient of MFCC & 79 & 97 & 97 & 84 & 39 & 55 & 17 & 66.86 \\
\hline 1st Coefficient of Chroma Vector & 71 & 86 & 86 & 98 & 26 & 48 & 53 & 66.86 \\
\hline Spectral Roll-off & 81 & 43 & 79 & 66 & 56 & 59 & 81 & 66.43 \\
\hline Spectral Crest & 61 & 80 & 60 & 26 & 92 & 71 & 75 & 66.43 \\
\hline 6th Coefficient of Chroma Vector & 100 & 76 & 99 & 31 & 61 & 45 & 52 & 66.29 \\
\hline 7th Coefficient of Chroma Vector & 37 & 99 & 98 & 1 & 96 & 42 & 86 & 65.57 \\
\hline Median Frequency & 88 & 35 & 78 & 71 & 102 & 78 & 2 & 64.86 \\
\hline 2nd Coefficient of Chroma Vector & 89 & 7 & 96 & 89 & 41 & 81 & 51 & 64.86 \\
\hline Spectral Centroid & 76 & 79 & 43 & 39 & 86 & 69 & 58 & 64.29 \\
\hline Difference Absolute Std. Dev. Value & 84 & 38 & 37 & 81 & 79 & 63 & 64 & 63.71 \\
\hline Shape Factor & 59 & 53 & 53 & 54 & 72 & 80 & 73 & 63.43 \\
\hline Spectral Mean & 43 & 77 & 77 & 96 & 46 & 40 & 61 & 62.86 \\
\hline Simple Square Integral & 4 & 72 & 72 & 22 & 89 & 89 & 92 & 62.86 \\
\hline 3rd Coefficient of GFCC & 95 & 29 & 88 & 94 & 34 & 53 & 45 & 62.57 \\
\hline 4th Coefficient of Chroma Vector & 40 & 96 & 30 & 87 & 85 & 52 & 47 & 62.43 \\
\hline Root Mean Square & 45 & 56 & 56 & 77 & 43 & 87 & 69 & 61.86 \\
\hline Signal to Noise Distortion & 97 & 69 & 69 & 47 & 74 & 31 & 42 & 61.29 \\
\hline 9th Coefficient of Chroma Vector & 72 & 12 & 95 & 88 & 27 & 76 & 57 & 61.00 \\
\hline Mean Absolute Deviation & 58 & 48 & 48 & 49 & 76 & 82 & 62 & 60.43 \\
\hline Root Squared 2nd Order Moment & 91 & 82 & 71 & 8 & 59 & 20 & 91 & 60.29 \\
\hline Root Squared 4th Order Moment & 101 & 71 & 82 & 82 & 4 & 66 & 14 & 60.00 \\
\hline 10th Coefficient of Chroma Vector & 36 & 95 & 85 & 28 & 73 & 41 & 56 & 59.14 \\
\hline 12th Coefficient of Chroma Vector & 90 & 89 & 89 & 59 & 10 & 37 & 38 & 58.86 \\
\hline 1st Coefficient of GFCC & 69 & 87 & 87 & 61 & 66 & 38 & 3 & 58.71 \\
\hline Mean & 80 & 26 & 26 & 80 & 80 & 68 & 44 & 57.71 \\
\hline Enhanced Mean Absolute Value & 21 & 73 & 73 & 62 & 58 & 54 & 63 & 57.71 \\
\hline Root Sum of Squares & 53 & 49 & 52 & 99 & 18 & 58 & 72 & 57.29 \\
\hline 2nd Coefficient of LTP & 82 & 70 & 70 & 35 & 36 & 12 & 95 & 57.14 \\
\hline Katz Fractal Dimension & 67 & 3 & 3 & 67 & 90 & 75 & 90 & 56.43 \\
\hline
\end{tabular}


Table 8. List of features extracted for method II and sorted with respect to MR value. Bold font indicates the top 24 ranked features.

\begin{tabular}{|c|c|c|c|c|c|c|c|c|}
\hline Feature & TT & KLD & BD & ROC & MWT & MRMR & RRF & MR \\
\hline 7th Coefficient of Chroma Vector & 100 & 98 & 98 & 26 & 96 & 97 & 55 & 81.43 \\
\hline 4th Coefficient of Chroma Vector & 89 & 96 & 76 & 73 & 85 & 52 & 96 & 81.00 \\
\hline Mobility & 93 & 64 & 64 & 67 & 68 & 91 & 95 & 77.43 \\
\hline Spectral Centroid & 68 & 78 & 78 & 77 & 45 & 98 & 88 & 76.00 \\
\hline Enhanced Mean Absolute Value & 71 & 95 & 95 & 28 & 98 & 56 & 87 & 75.71 \\
\hline 9th Coefficient of LTP & 64 & 101 & 101 & 64 & 93 & 79 & 19 & 74.43 \\
\hline 1st Coefficient of GFCC & 95 & 35 & 97 & 98 & 38 & 99 & 54 & 73.71 \\
\hline 7th Coefficient of LTP & 57 & 93 & 93 & 57 & 101 & 24 & 89 & 73.43 \\
\hline Slope Sign Change & 74 & 73 & 89 & 79 & 89 & 89 & 13 & 72.29 \\
\hline Maximum Fractal Length & 3 & 72 & 74 & 91 & 90 & 90 & 70 & 70.00 \\
\hline 3rd Coefficient of MFCC & 38 & 97 & 22 & 95 & 102 & 80 & 53 & 69.57 \\
\hline 6th Coefficient of Chroma Vector & 69 & 80 & 96 & 90 & 27 & 68 & 56 & 69.43 \\
\hline 8th Coefficient of Chroma Vector & 61 & 99 & 99 & 69 & 66 & 49 & 40 & 69.00 \\
\hline Enhanced Wavelength & 81 & 85 & 85 & 68 & 94 & 16 & 50 & 68.43 \\
\hline Pulse Percentage Rate & 4 & 94 & 100 & 42 & 73 & 76 & 77 & 66.57 \\
\hline Root Squared Zero Order Moment & 63 & 84 & 84 & 30 & 69 & 72 & 46 & 64.00 \\
\hline Crest Factor & 51 & 68 & 68 & 80 & 72 & 32 & 72 & 63.29 \\
\hline Modified Mean Absolute Value 2 & 42 & 90 & 90 & 7 & 88 & 64 & 62 & 63.29 \\
\hline Spectral Crest & 87 & 58 & 45 & 40 & 47 & 85 & 75 & 62.43 \\
\hline 2nd Coeffient of MFCC & 37 & 69 & 69 & 84 & 77 & 48 & 51 & 62.14 \\
\hline 1st Coeffient of MFCC & 77 & 9 & 9 & 100 & 99 & 38 & 102 & 62.00 \\
\hline Average Frequency & 80 & 29 & 54 & 47 & 54 & 86 & 82 & 61.71 \\
\hline 4th Coefficient of LTP & 34 & 65 & 65 & 65 & 42 & 74 & 84 & 61.29 \\
\hline Willison Amplitude & 91 & 74 & 72 & 15 & 16 & 77 & 83 & 61.14 \\
\hline Spectral Spread & 99 & 47 & 58 & 85 & 60 & 9 & 68 & 60.86 \\
\hline 3rd Coefficient of LTP & 65 & 91 & 91 & 5 & 65 & 2 & 101 & 60.00 \\
\hline Root Squared 4th Order Moment & 70 & 66 & 71 & 14 & 97 & 62 & 36 & 59.43 \\
\hline Lyapunov Exponent & 32 & 63 & 63 & 8 & 62 & 81 & 100 & 58.43 \\
\hline 2nd Coeffient of GFCC & 40 & 87 & 87 & 89 & 40 & 50 & 14 & 58.14 \\
\hline 3rd Coeffient of GFCC & 73 & 22 & 10 & 66 & 39 & 96 & 99 & 57.86 \\
\hline Correlation Dimension & 14 & 70 & 70 & 101 & 57 & 1 & 91 & 57.71 \\
\hline Root Squared 2nd Order Moment & 101 & 5 & 66 & 36 & 31 & 100 & 61 & 57.14 \\
\hline 5th Coefficient of Chroma Vector & 20 & 76 & 80 & 97 & 4 & 36 & 86 & 57.00 \\
\hline 2nd Coefficient of Chroma Vector & 75 & 41 & 41 & 88 & 78 & 73 & 2 & 56.86 \\
\hline 11th Coefficient of Chroma Vector & 66 & 39 & 39 & 74 & 95 & 47 & 37 & 56.71 \\
\hline Log Energy & 45 & 75 & 52 & 53 & 19 & 88 & 60 & 56.00 \\
\hline 10th Coefficient of Chroma Vector & 90 & 38 & 38 & 19 & 100 & 69 & 38 & 56.00 \\
\hline 5th Coefficient of LTP & 8 & 82 & 82 & 83 & 5 & 101 & 30 & 55.86 \\
\hline 1st Coefficient of LTP & 11 & 92 & 92 & 24 & 83 & 57 & 31 & 55.71 \\
\hline
\end{tabular}

\subsubsection{Feature Reduction Using Kernel PCA}

PCA applies orthogonal transformation to transform a group of likely correlated features into a set of linearly independent features known as principal components. These principal components represent the normalized linear combinations of the original features. It includes information about the most powerful variations present in the data set. The first principal component holds maximum variance information of the data set.

Kernel PCA (KPCA) [61,62] enhances the original PCA to non-linear data distribution problems through a kernel function. A kernel function projects low-dimensional feature data to a higher-dimensional feature space, where it becomes linearly separable [63]. 
The previous stage of hybrid feature selection reduced the feature dimensions to $1 \times 24$ which are fed to KPCA to further decrease dimensions for both methods I and II. Components of KPCA were selected recursively based on the classification performance through tenfold cross-validation. Separate sets of 5, 7, 10,12, 15, and 17 components were picked for methods I and II to investigate the classification performance for differentiating normal and hypertension signal classes of PuPG signals.

\subsection{Classification}

To perform the classification of normal and hypertension classes of PuPG signal data set, this study employed a range of classification methods through tenfold cross-validation schemes. The classification methods opted in this study were SVM-Linear (SVM-L), SVM-Quadratic (SVM-Q), SVM-Cubic (SVM-C), SVM-Fine Gaussian (SVM-FG), SVMMedium Gaussian (SVM-MG), KNN-Fine (KNN-F), KNN-Medium (KNN-M), KNN-Cosine distance (KNN-Cos), KNN-Cubic (KNN-C), KNN-Weighted (KNN-W), Decision Trees (DT), Linear Discriminant (LD), Logistic Regression (LR), Gaussian Naive Baise (NBG), Kernel Naive Baise (NBK), Ensemble Boosted Trees (Eboost), Ensemble Bagged Trees (EBT), Ensemble Subspace Discriminant (ESD), and Ensemble Subspace KNN (ESKNN). The tenfold cross-validation was also compared with 5-, 15-, and 20-fold cross-validation and $80-20 \%$ and $75-25 \%$ train-test experiments. All experiments were implemented on MATLAB 2018a on a personal computer with Core i7 with 32 GB RAM.

\section{Results}

In this study, the PuPG signal data set comprising two classes (Normal and Hypertension) was first preprocessed through DWT and EMD to develop methods I and II respectively. We obtained 102 features for each method, i.e., DWT and EMD. These features were subjected to the HFSR framework to reduce the computational complexity and feature vector dimensions. Standard statistical parameters of Accuracy (Acc), Sensitivity (Sen), Specificity (Sp), and Error rate (Err) were used to measure the classification performance.

\subsection{Method I}

In this research, a comparative analysis was performed via preprocessing the PuPG signal through DWT and EMD. This section presents the results yielded by preprocessing through DWT and succeeding processes of feature extraction, selection, and classification. Various feature sets, namely $S_{1}, S_{2}, S_{3}, S_{4}, S_{5}$, and $S_{6}$ were formed by randomly choosing $5,7,10,12,15$, and 17 transformed features. These feature components were fed to several classification methods to examine the diagnostic performance through tenfold crossvalidation. Table 9 presents consolidated result analysis of various classification methods for features sets $S_{1}$ (5 components), $S_{2}$ (7 components), and $S_{3}$ (10 components). Table 10 illustrates comprehensive analysis of classification performance over different classifiers for feature sets $S_{4}$ (12 components), $S_{5}$ (15 components), and $S_{6}$ (17 components). As expressed in Table 10, Ensemble Subspace KNN classifier scores highest average accuracy of $98.4 \%$, for 12 feature components, i.e., $S_{4}$ feature set.

Figure 11 shows the performance in terms of accuracy for different feature sets in various classifiers for distinguishing normal and hypertension classes using PuPG signals. Figure 12 demonstrates the specificity performance of several classifiers for various features sets from DWT based preprocessing method. Figure 13 presents a graphical comparison of the sensitivity performance of several classifiers for different feature combinations.

NBG classifier achieves highest specificity performance of $100 \%$ for feature sets $S_{3}$, $S_{4}, S_{5}$, and $S_{6}$ (Figure 12), but it reaches maximum sensitivities of $26 \%, 26 \%, 32 \%$, and $34 \%$ for the same feature sets (Figure 13); therefore, it results in significant reduction of overall classifier accuracy of NBG. The sensitivity performance is $100 \%$ for several classifiers (LD, LR, NBG, SVM-FG, SVM-MG, EBT) for feature set $S_{1}$ (Figure 13), but the specificity performance is comparatively low. 
Table 9. Consolidated result analysis of feature sets $\left(S_{1}, S_{2}, S_{3}\right)$ for method I with various classifiers.

\begin{tabular}{cccccccccccccc}
\hline \multirow{2}{*}{ Classifier } & \multicolumn{4}{c}{$\boldsymbol{S}_{\mathbf{1}}$ (5 Components) } & \multicolumn{4}{c}{$\boldsymbol{S}_{\mathbf{2}}$ (7 Components) } & \multicolumn{3}{c}{$S_{\mathbf{3}}$ (10 Components) } \\
\cline { 2 - 5 } & Acc & Sp & Sen & Err & Acc & Sp & Sen & Err & Acc & Sp & Sen & Err \\
\hline DT & 0.874 & 0.89 & 0.89 & 0.126 & 0.924 & 0.92 & 0.93 & 0.076 & 0.934 & 0.94 & 0.93 & 0.066 \\
LD & 0.688 & 0.3 & 1 & 0.312 & 0.688 & 0.3 & 1 & 0.312 & 0.669 & 0.3 & 0.97 & 0.331 \\
LR & 0.688 & 0.3 & 1 & 0.312 & 0.688 & 0.3 & 1 & 0.312 & 0.688 & 0.3 & 1 & 0.312 \\
NBG & 0.688 & 0.3 & 1 & 0.312 & 0.688 & 0.3 & 1 & 0.312 & 0.59 & 1 & 0.26 & 0.41 \\
NBK & 0.804 & 0.91 & 0.72 & 0.196 & 0.83 & 0.92 & 0.76 & 0.17 & 0.893 & 0.91 & 0.88 & 0.107 \\
SVM-L & 0.479 & 0.56 & 0.41 & 0.521 & 0.587 & 0.11 & 0.97 & 0.413 & 0.498 & 0.37 & 0.6 & 0.502 \\
SVM-Q & 0.527 & 0.65 & 0.43 & 0.473 & 0.527 & 0.08 & 0.89 & 0.473 & 0.546 & 0.41 & 0.65 & 0.454 \\
SVM-C & 0.47 & 0.4 & 0.53 & 0.53 & 0.555 & 0.03 & 0.98 & 0.445 & 0.524 & 0 & 0.94 & 0.476 \\
SVM-FG & 0.688 & 0.3 & 1 & 0.312 & 0.688 & 0.30 & 1 & 0.312 & 0.688 & 0.3 & 1 & 0.312 \\
SVM-MG & 0.688 & 0.3 & 1 & 0.312 & 0.688 & 0.30 & 1 & 0.312 & 0.688 & 0.3 & 1 & 0.312 \\
KNN-F & 0.937 & 0.9 & 0.97 & 0.063 & 0.972 & 0.96 & 0.98 & 0.028 & 0.984 & 0.97 & 0.99 & 0.016 \\
KNN-M & 0.792 & 0.68 & 0.88 & 0.208 & 0.864 & 0.81 & 0.91 & 0.136 & 0.905 & 0.86 & 0.94 & 0.095 \\
KNN-Cos & 0.685 & 0.3 & 0.99 & 0.315 & 0.681 & 0.3 & 0.99 & 0.319 & 0.685 & 0.3 & 0.99 & 0.315 \\
KNN-C & 0.672 & 0.68 & 0.66 & 0.328 & 0.871 & 0.83 & 0.9 & 0.129 & 0.896 & 0.84 & 0.94 & 0.104 \\
KNN-W & 0.921 & 0.88 & 0.95 & 0.079 & 0.965 & 0.96 & 0.97 & 0.035 & 0.978 & 0.97 & 0.98 & 0.022 \\
Eboost & 0.918 & 0.89 & 0.94 & 0.082 & 0.864 & 0.74 & 0.96 & 0.136 & 0.555 & 0 & 1 & 0.445 \\
EBT & 0.688 & 0.3 & 1 & 0.312 & 0.972 & 0.95 & 0.99 & 0.028 & 0.943 & 0.93 & 0.95 & 0.057 \\
ESD & 0.94 & 0.92 & 0.95 & 0.06 & 0.688 & 0.3 & 1 & 0.312 & 0.681 & 0.3 & 0.99 & 0.319 \\
ESKNN & 0.915 & 0.91 & 0.91 & 0.085 & 0.984 & 0.98 & 0.99 & 0.016 & 0.981 & 0.97 & 0.99 & 0.019 \\
\hline
\end{tabular}

Table 10. Consolidated result analysis of feature sets $\left(S_{4}, S_{5}, S_{6}\right)$ for method I with various classifiers. Bold font indicates best results.

\begin{tabular}{|c|c|c|c|c|c|c|c|c|c|c|c|c|}
\hline \multirow{2}{*}{ Classifier } & \multicolumn{4}{|c|}{$S_{4}$ (12 Components) } & \multicolumn{4}{|c|}{$S_{5}$ (15 Components) } & \multicolumn{4}{|c|}{$S_{6}$ (17 Components) } \\
\hline & Acc & $\mathrm{Sp}$ & Sen & Err & Acc & Sp & Sen & Err & Acc & $S p$ & Sen & Err \\
\hline DT & 0.959 & 0.96 & 0.96 & 0.041 & 0.959 & 0.94 & 0.98 & 0.041 & 0.972 & 0.96 & 98 & 0.028 \\
\hline LD & 0.581 & 0.3 & 0.99 & 0.419 & 0.662 & 0.3 & 0.95 & 0.338 & 0.691 & 0.32 & 0.99 & 0.309 \\
\hline LR & 0.688 & 0.3 & 1 & 0.312 & 0.688 & 0.3 & 1 & 0.312 & 0.675 & 0.35 & 0.94 & 0.325 \\
\hline NBG & 0.59 & 1 & 0.26 & 0.41 & 0.625 & 1 & 0.32 & 0.375 & 0.631 & 1 & 0.34 & 0.369 \\
\hline NBK & 0.868 & 0.85 & 0.89 & 0.132 & 0.877 & 0.92 & 0.84 & 0.123 & 0.88 & 0.91 & 0.95 & 0.12 \\
\hline SVM-L & 0.524 & 0.26 & 0.74 & 0.476 & 0.543 & 0.12 & 0.88 & 0.457 & 0.536 & 0.07 & 0.91 & 0.464 \\
\hline SVM-Q & 0.552 & 0.22 & 0.82 & 0.448 & 0.536 & 0.33 & 0.7 & 0.464 & 0.546 & 0.02 & 0.97 & 0.454 \\
\hline SVM-C & 0.524 & 0.8 & 0.88 & 0.476 & 0.517 & 0 & 0.93 & 0.483 & 0.514 & 0.05 & 0.89 & 0.486 \\
\hline SVM-FG & 0.688 & 0.3 & 1 & 0.312 & 0.688 & 0.3 & 1 & 0.312 & 0.7 & 0.33 & 1 & 0.3 \\
\hline SVM-MG & 0.665 & 0.3 & 1 & 0.335 & 0.688 & 0.3 & 1 & 0.312 & 0.694 & 0.31 & 1 & 0.306 \\
\hline KNN-F & 0.981 & 0.97 & 0.99 & 0.019 & 0.975 & 0.97 & 0.98 & 0.025 & 0.915 & 0.88 & 0.94 & 0.085 \\
\hline KNN-M & 0.918 & 0.85 & 0.97 & 0.082 & 0.912 & 0.85 & 0.96 & 0.088 & 0.659 & 0.7 & 0.63 & 0.341 \\
\hline KNN-Cos & 0.688 & 0.3 & 1 & 0.312 & 0.688 & 0.3 & 1 & 0.312 & 0.685 & 0.3 & 0.99 & 0.315 \\
\hline KNN-C & 0.905 & 0.85 & 0.95 & 0.095 & 0.909 & 0.85 & 0.95 & 0.091 & 0.909 & 0.89 & 0.93 & 0.091 \\
\hline KNN-W & 0.981 & 0.98 & 0.98 & 0.019 & 0.975 & 0.97 & 0.98 & 0.025 & 0.978 & 0.97 & 0.98 & 0.022 \\
\hline Eboost & 0.555 & 0 & 1 & 0.445 & 0.555 & 0 & 1 & 0.445 & 0.555 & 0 & 1 & 0.445 \\
\hline EBT & 0.965 & 0.94 & 0.98 & 0.035 & 0.972 & 0.97 & 0.97 & 0.028 & 0.94 & 0.92 & 0.95 & 0.06 \\
\hline ESD & 0.688 & 0.3 & 1 & 0.312 & 0.666 & 0.3 & 0.96 & 0.334 & 0.681 & 0.3 & 0.99 & 0.319 \\
\hline ESKNN & 0.984 & 0.97 & 0.99 & 0.016 & 0.975 & 0.97 & 0.98 & 0.025 & 0.981 & 0.99 & 0.99 & 0.019 \\
\hline
\end{tabular}




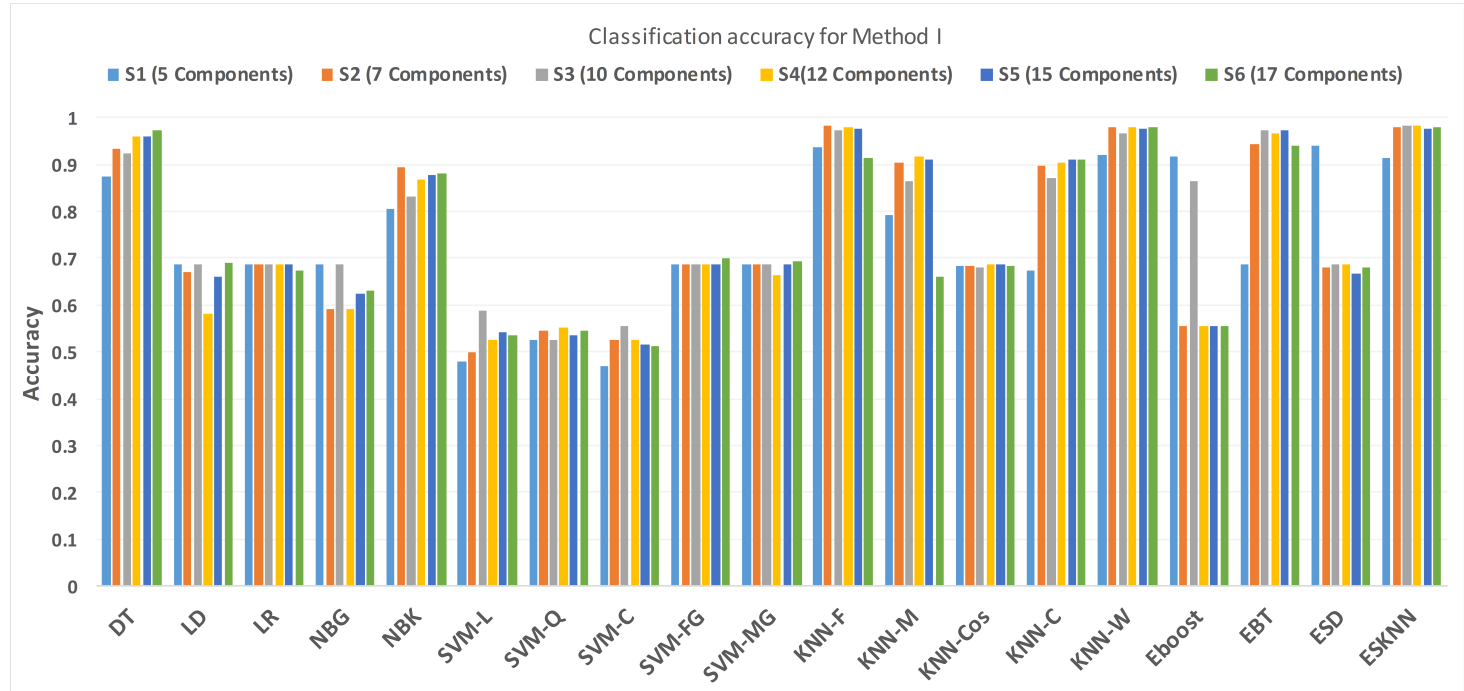

Figure 11. Performance of accuracy for different feature sets in various classifiers for PuPG signal through method I.

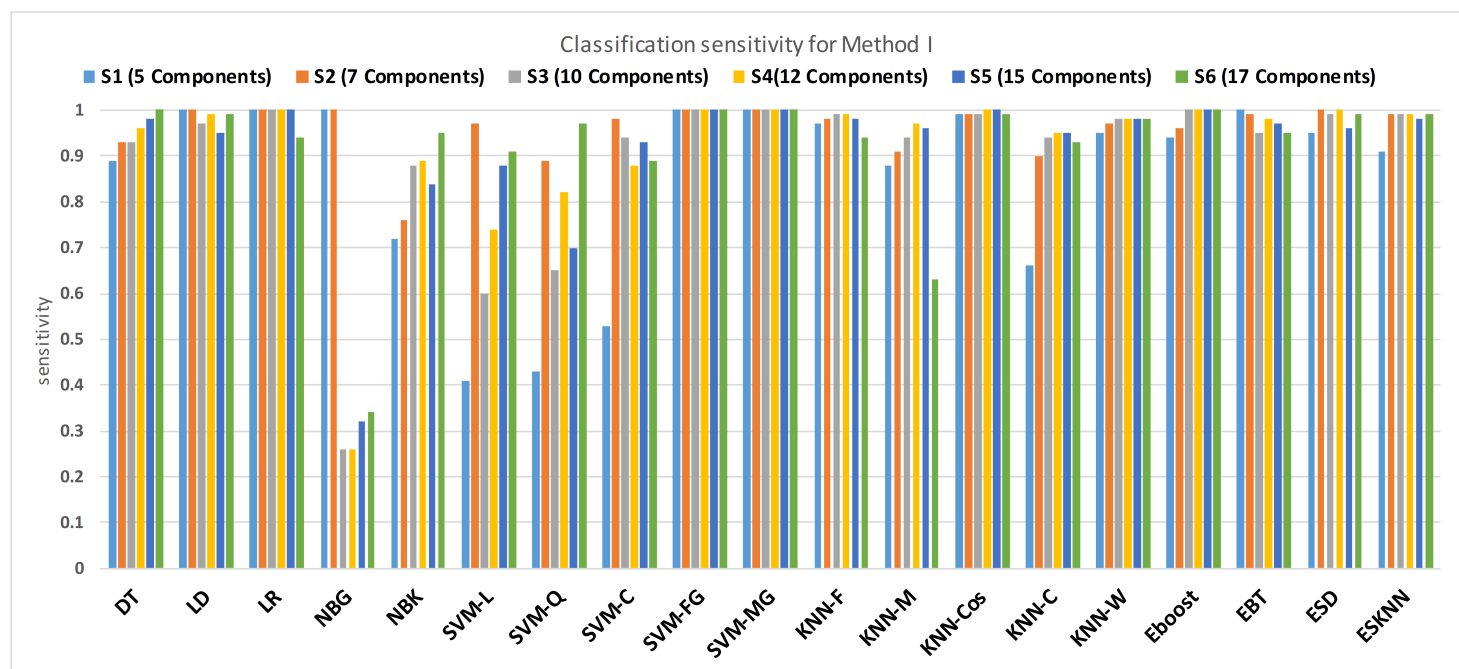

Figure 12. Performance of sensitivity for different feature sets in various classifiers for PuPG signal through method I.

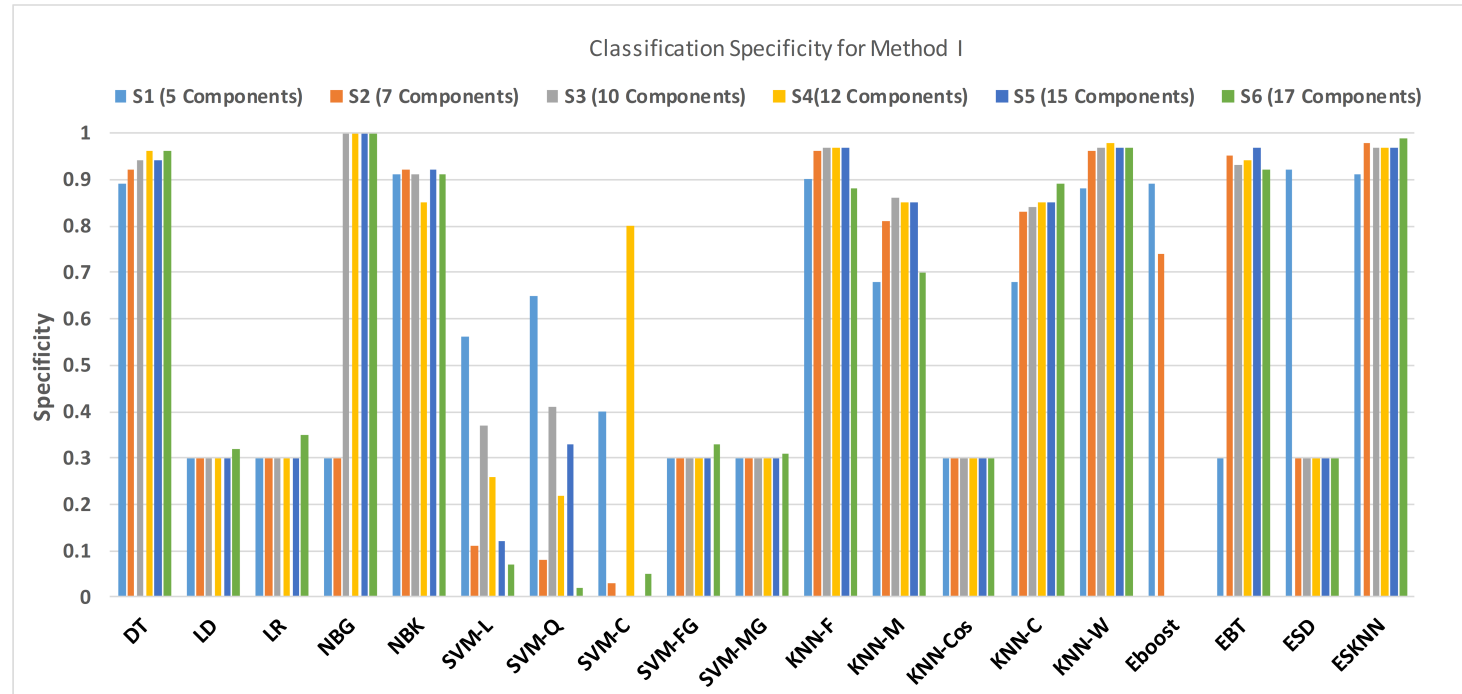

Figure 13. Performance of specificity for different feature sets in various classifiers for PuPG signal through method I. 
Figure 14 shows the classification performance results in the form of a confusion matrix for best configurations such as ESKNN classifier with $S_{4}$ (12 feature components). The sensitivity of classification is $99 \%$, which means that out of 700 PuPG signals of hypertension, 693 were correctly predicted as hypertension data class while testing, whereas only seven were misclassified as healthy class. The classifier achieved a $98 \%$ specificity performance. Out of 709 healthy PuPG signal samples, 695 were accurately predicted as healthy class, whereas the remaining 14 signals were misclassified.

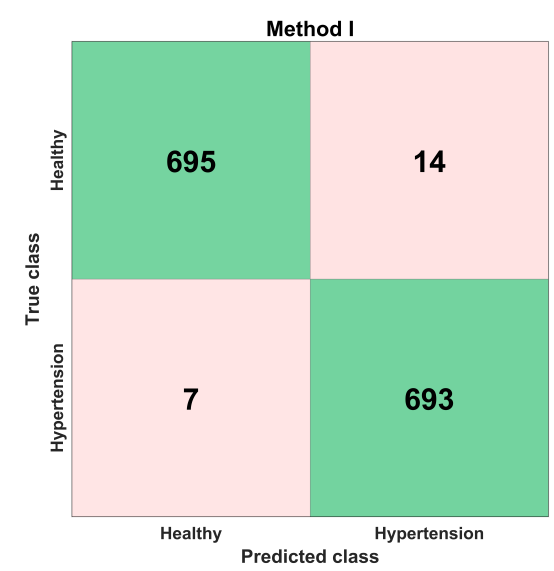

(a) Confusion matrix in terms of numbers

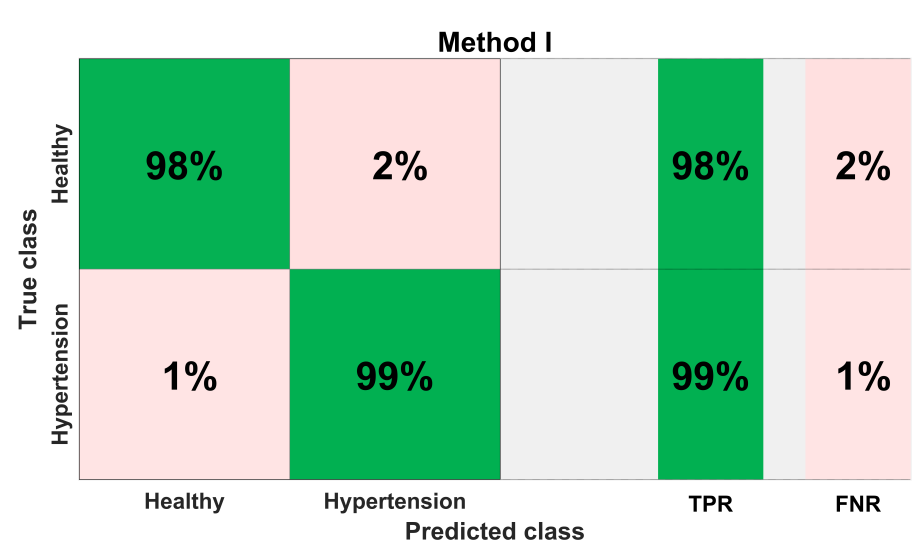

(b) Confusion matrix in terms of percentage

Figure 14. Confusion matrix for method I.

Table 11 includes the extensive experimentation results to avoid the classifier overfitting. The selected configuration was tested through 5-, 10-, 15-, and 20-fold cross-validation and $20 \%$ and $25 \%$ train-test holdout validations.

Table 11. Validation of the selected scheme of method I.

\begin{tabular}{ccccc}
\hline Evaluation & Classes & Accuracy & True Positive Rate & False Negative Rate \\
\hline \multirow{2}{*}{ 5-Fold Cross-Validation } & Healthy & \multirow{2}{*}{0.983} & 0.98 & 0.02 \\
& Hypertension & & 0.99 & 0.01 \\
\hline \multirow{2}{*}{ 10-Fold Cross-Validation } & Healthy & \multirow{2}{*}{0.984} & 0.98 & 0.02 \\
& Hypertension & & 0.99 & 0.01 \\
\hline \multirow{2}{*}{ 15-Fold Cross-Validation } & Healthy & \multirow{2}{*}{0.984} & 0.98 & 0.02 \\
& Hypertension & \multirow{2}{*}{0.984} & 0.99 & 0.01 \\
\hline \multirow{2}{*}{ 20-Fold Cross-Validation } & Healthy & 0.99 & 0.02 \\
& Hypertension & \multirow{2}{*}{0.978} & 0.01 \\
\hline \multirow{2}{*}{ 20\% Hold Out Validation } & Healthy & 0.94 & 0 \\
& Hypertension & 0.98 & 0.06 \\
\hline \multirow{2}{*}{ 25\% Hold Out Validation } & Healthy & \multirow{2}{*}{0.989} & 1 & 0.02 \\
\hline
\end{tabular}

\subsection{Method II}

This section is primarily focused on the second method that is under discussion for this research. It encompasses the results of the classification of the features extracted after the preprocessing of the PuPG signal via EMD. A certain number of feature sets were chosen that were the result of the HFSR. The feature sets comprising of 5, 7, 10, 12, 15, and 17 transformed features were chosen and named $S_{1}, S_{2}, S_{3}, S_{4}, S_{5}$, and $S_{6}$, respectively. These feature components were fed to a various number of classifiers for classification and their performance was tested through tenfold cross-validation. 
Table 12 depicts the results obtained after the classification of the feature set $S_{1}$ (5 components), $S_{2}$ (7 components), and $S_{3}$ (10 components) on using a selection of various classifiers. Table 13 shows the outcomes of various classification techniques applied on feature sets $S_{4}$ (12 components), $S_{5}$ (15 components), and $S_{6}$ (17 components). Analysis of both Tables 12 and 13 show that a maximum average accuracy using the least number of features is $99.4 \%$. This accuracy is the result of the weighted KNN classification method applied on the feature set $S_{1}$.

Table 12. Feature analysis table $\left(S_{1}, S_{2}, S_{3}\right)$ for method II. Bold font indicates the best results.

\begin{tabular}{|c|c|c|c|c|c|c|c|c|c|c|c|c|}
\hline \multirow{2}{*}{ Classifier } & \multicolumn{4}{|c|}{$S_{1}$ (5 Components) } & \multicolumn{4}{|c|}{$S_{2}$ (7 Components) } & \multicolumn{4}{|c|}{$S_{3}$ (10 Components) } \\
\hline & Acc & $S p$ & Sen & Err & Acc & $\mathrm{Sp}$ & Sen & Err & Acc & $\mathrm{Sp}$ & Sen & Err \\
\hline DT & 0.974 & 0.98 & 0.97 & 0.026 & 0.983 & 0.98 & 0.99 & 0.017 & 0.989 & 0.98 & 0.99 & 0.011 \\
\hline LD & 0.619 & 0.24 & 1 & 0.381 & 0.619 & 0.24 & 1 & 0.381 & 0.568 & 0.24 & 0.9 & 0.432 \\
\hline LR & 0.679 & 0.97 & 0.39 & 0.321 & 0.679 & 0.97 & 0.39 & 0.321 & 0.679 & 0.97 & 0.39 & 0.321 \\
\hline NBG & 0.619 & 0.24 & 1 & 0.381 & 0.619 & 0.24 & 1 & 0.381 & 0.268 & 1 & 0.26 & 0.732 \\
\hline NBK & 0.946 & 0.97 & 0.92 & 0.054 & 0.946 & 0.98 & 0.91 & 0.054 & 0.94 & 0.97 & 0.91 & 0.06 \\
\hline SVM-L & 0.48 & 0.49 & 0.47 & 0.52 & 0.497 & 0.59 & 0.41 & 0.503 & 0.523 & 0.52 & 0.53 & 0.477 \\
\hline SVM-Q & 0.51 & 0.53 & 0.5 & 0.49 & 0.511 & 0.24 & 0.78 & 0.489 & 0.497 & 0.46 & 0.53 & 0.503 \\
\hline SVM-C & 0.49 & 0.3 & 0.69 & 0.51 & 0.491 & 0.22 & 0.77 & 0.509 & 0.491 & 0.23 & 0.76 & 0.509 \\
\hline SVM-FG & 0.668 & 1 & 0.34 & 0.332 & 0.662 & 1 & 0.32 & 0.338 & 0.665 & 0.99 & 0.34 & 0.335 \\
\hline SVM-MG & 0.619 & 0.24 & 1 & 0.381 & 0.614 & 0.51 & 0.72 & 0.386 & 0.597 & 0.73 & 0.46 & 0.403 \\
\hline KNN-F & 0.99 & 0.99 & 0 & 0.01 & 0.893 & 0.98 & 0.984 & 0.107 & 0.991 & 0.99 & 0.99 & 0.009 \\
\hline KNN-M & 0.957 & 0.93 & 0.98 & 0.043 & 0.969 & 0.95 & 0.99 & 0.031 & 0.972 & 0.95 & 0.99 & 0.028 \\
\hline KNN-Cos & 0.631 & 0.27 & 0.99 & 0.369 & 0.639 & 0.3 & 0.98 & 0.361 & 0.636 & 0.3 & 0.98 & 0.364 \\
\hline KNN-C & 0.957 & 0.93 & 0.98 & 0.043 & 0.966 & 0.94 & 0.99 & 0.034 & 0.969 & 0.94 & 0.99 & 0.031 \\
\hline KNN-W & 0.994 & 0.992 & 0.996 & 0.006 & 0.986 & 0.94 & 0.99 & 0.014 & 0.992 & 0.99 & 0.99 & 0.008 \\
\hline Eboost & 0.489 & 0.39 & 0.59 & 0.511 & 0.489 & 0.39 & 0.59 & 0.511 & 0.489 & 0.39 & 0.59 & 0.511 \\
\hline EBT & 0.98 & 0.97 & 0.99 & 0.02 & 0.986 & 0.98 & 0.99 & 0.014 & 0.986 & 0.98 & 0.99 & 0.014 \\
\hline ESD & 0.619 & 0.24 & 1 & 0.381 & 0.619 & 0.24 & 1 & 0.381 & 0.571 & 0.24 & 0.9 & 0.429 \\
\hline ESKNN & 0.991 & 0.99 & 0.99 & 0.009 & 0.983 & 0.99 & 0.98 & 0.017 & 0.991 & 0.99 & 0.99 & 0.009 \\
\hline
\end{tabular}

Table 13. Feature analysis table $\left(S_{4}, S_{5}, S_{6}\right)$ for method II.

\begin{tabular}{cccccccccccccc}
\hline \multirow{2}{*}{ Classifier } & \multicolumn{4}{c}{$\boldsymbol{S}_{\mathbf{4}} \mathbf{( 1 2}$ Components) } & \multicolumn{4}{c}{$\boldsymbol{S}_{\mathbf{5}}$ (15 Components) } & \multicolumn{3}{c}{$\boldsymbol{S}_{\mathbf{6}}$ (17 Components) } \\
\cline { 2 - 5 } & Acc & Sp & Sen & Err & Acc & Sp & Sen & Err & Acc & Sp & Sen & Err \\
\hline DT & 0.992 & 0.99 & 0.99 & 0.008 & 0.972 & 0.95 & 0.99 & 0.028 & 0.983 & 0.98 & 0.98 & 0.017 \\
LD & 0.548 & 0.32 & 0.78 & 0.452 & 0.565 & 0.34 & 0.8 & 0.435 & 0.665 & 1 & 0.33 & 0.335 \\
LR & 0.679 & 0.97 & 0.39 & 0.321 & 0.679 & 0.97 & 0.39 & 0.321 & 0.676 & 0.97 & 0.38 & 0.324 \\
NBG & 0.636 & 0.97 & 0.39 & 0.364 & 0.662 & 1 & 0.32 & 0.338 & 0.665 & 1 & 0.33 & 0.335 \\
NBK & 0.92 & 0.96 & 0.88 & 0.08 & 0.926 & 0.97 & 0.89 & 0.074 & 0.909 & 0.95 & 0.87 & 0.091 \\
SVM-L & 0.531 & 0.27 & 0.79 & 0.469 & 0.486 & 0.23 & 0.74 & 0.514 & 0.5 & 0.24 & 0.76 & 0.5 \\
SVM-Q & 0.503 & 0.19 & 0.82 & 0.497 & 0.469 & 0.15 & 0.79 & 0.531 & 0.514 & 0.15 & 0.88 & 0.486 \\
SVM-C & 0.472 & 0 & 0.94 & 0.528 & 0.472 & 0.1 & 0.85 & 0.528 & 0.486 & 0 & 0.97 & 0.514 \\
SVM-F & 0.662 & 1 & 0.32 & 0.338 & 0.662 & 1 & 0.32 & 0.338 & 0.696 & 1 & 0.39 & 0.304 \\
SVM-MG & 0.665 & 1 & 0.33 & 0.335 & 0.662 & 1 & 0.32 & 0.338 & 0.696 & 1 & 0.39 & 0.304 \\
KNN-F & 0.991 & 0.99 & 0.99 & 0.009 & 0.983 & 0.98 & 0.98 & 0.017 & 0.989 & 0.98 & 0.99 & 0.011 \\
KNN-M & 0.949 & 0.94 & 0.96 & 0.051 & 0.96 & 0.94 & 0.98 & 0.04 & 0.94 & 0.97 & 0.91 & 0.06 \\
KNN-Cos & 0.639 & 0.28 & 1 & 0.361 & 0.628 & 0.28 & 0.97 & 0.372 & 0.645 & 0.3 & 0.99 & 0.355 \\
KNN-C & 0.946 & 0.94 & 0.95 & 0.054 & 0.963 & 0.94 & 0.98 & 0.037 & 0.94 & 0.98 & 0.9 & 0.06 \\
KNN-W & 0.991 & 0.99 & 0.99 & 0.009 & 0.986 & 0.99 & 0.98 & 0.014 & 0.993 & 0.99 & 0.99 & 0.007 \\
Eboost & 0.489 & 0.39 & 0.59 & 0.511 & 0.534 & 0.48 & 0.59 & 0.466 & 0.489 & 0.39 & 0.59 & 0.511 \\
EBT & 0.989 & 0.98 & 0.99 & 0.011 & 0.966 & 0.97 & 0.96 & 0.034 & 0.986 & 0.99 & 0.98 & 0.014 \\
ESD & 0.577 & 0.26 & 0.89 & 0.423 & 0.563 & 0.39 & 0.73 & 0.437 & 0.665 & 1 & 0.33 & 0.335 \\
ESKNN & 0.991 & 0.99 & 0.99 & 0.009 & 0.983 & 0.98 & 0.98 & 0.017 & 0.991 & 0.99 & 0.99 & 0.009 \\
\hline
\end{tabular}


Figure 15 shows a comparison of the performance of various classifiers based on the accuracy achieved as a result of distinguishing hypertension and normal PuPG signal. Figure 16 depicts the comparison result of various classifiers based on their specificities after using EMD as the preprocessing technique. Figure 17 represents the comparison of the sensitivities of various classification methods. NBG classifier achieves the highest specificity performance of $100 \%$ for feature sets $S_{3}$ (Figure 16), but it reaches maximum sensitivities of $26 \%$ for the same feature set (Figure 17). The sensitivity performance is $100 \%$ for several classifiers (LD, NBG, SVM-MG, ESD) for feature set $S_{1}$ (Figure 17), but the specificity performance is comparatively low.

Figure 18 illustrates the best classification performance in the form of a confusion matrix for selected features set $\left(S_{1}\right)$ with $\mathrm{KNN}-\mathrm{W}$ classifier. The sensitivity of classification is more than $99 \%$, which means only one out of 700 PuPG signals was wrong predicted as hypertension data class, whereas the remaining 699 PuPG signals were correctly identified as hypertension. Out of 709 healthy PuPG signals, 702 were correctly predicted as healthy, achieving specificity of $99 \%$. The overall average classification accuracy in the best configuration with the KNN-W classifier was $99.4 \%$.

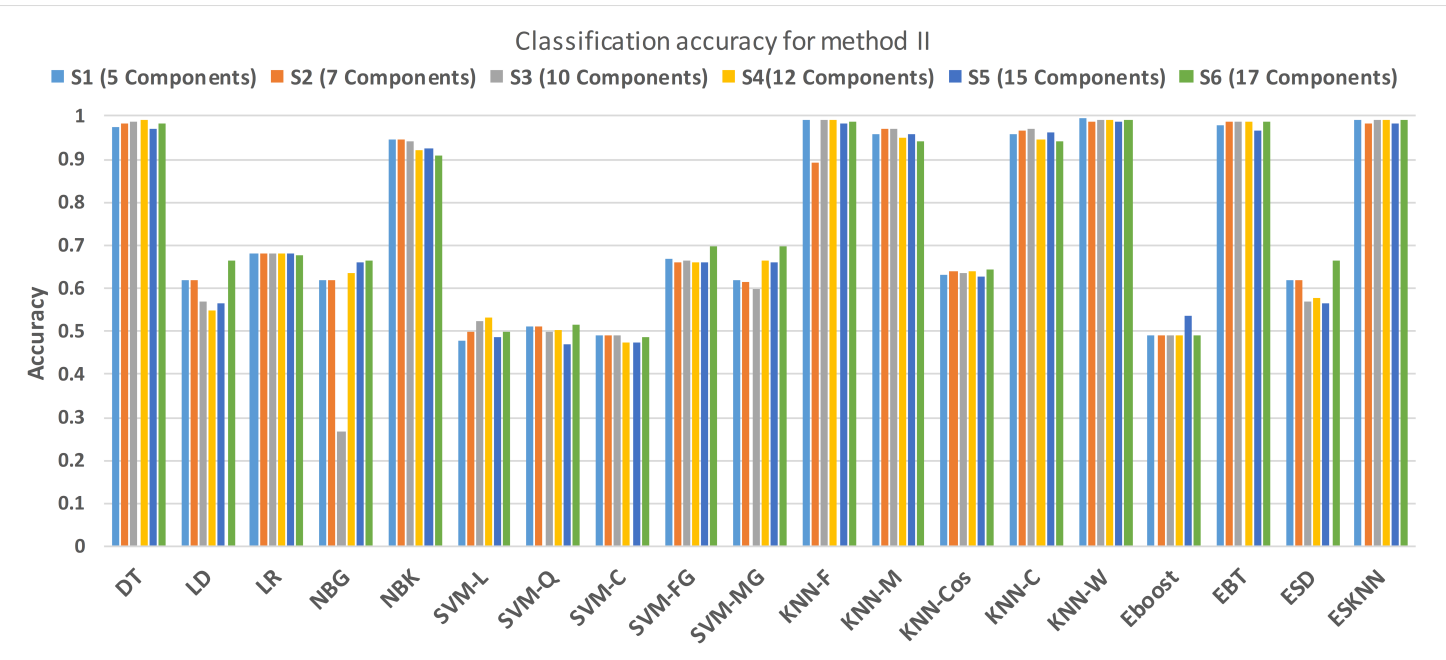

Figure 15. Performance of accuracy for different feature sets in various classifiers for PuPG signal through method II.

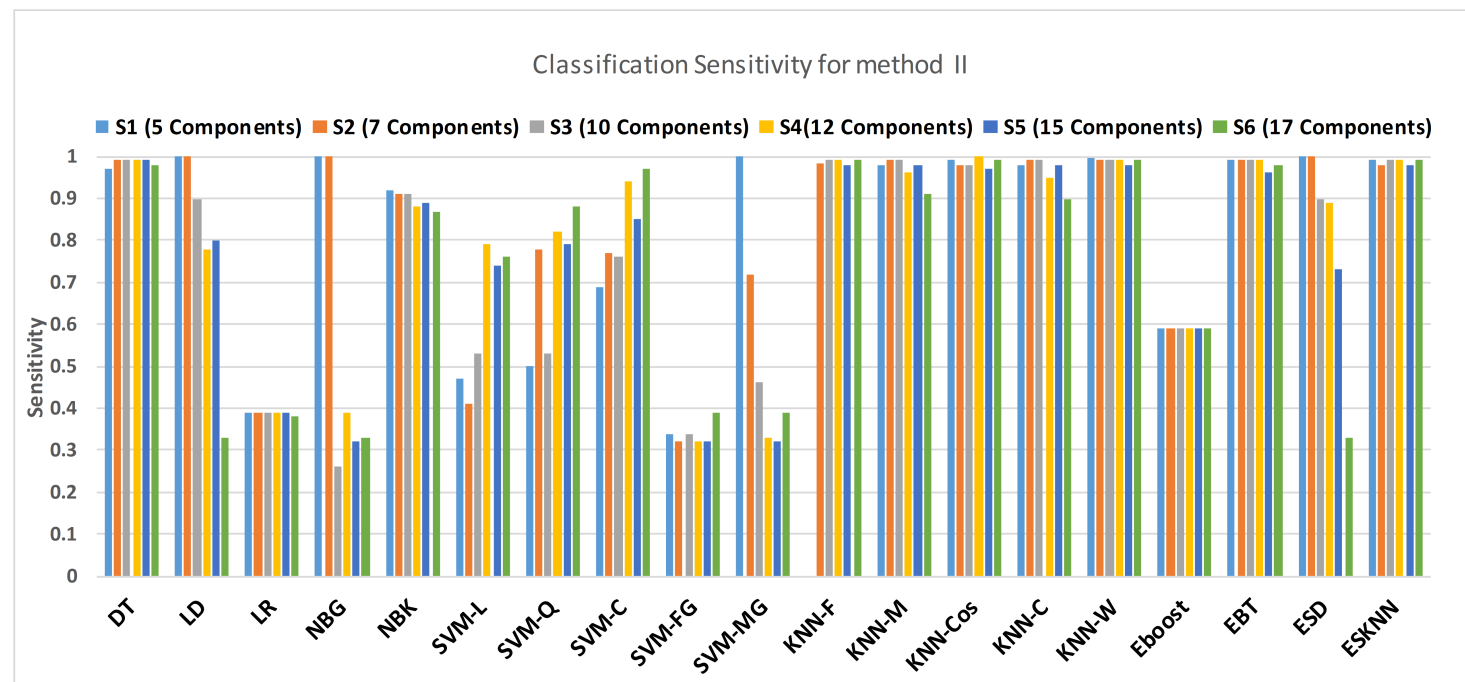

Figure 16. Performance of sensitivity for different feature sets in various classifiers for PuPG signal through method II. 


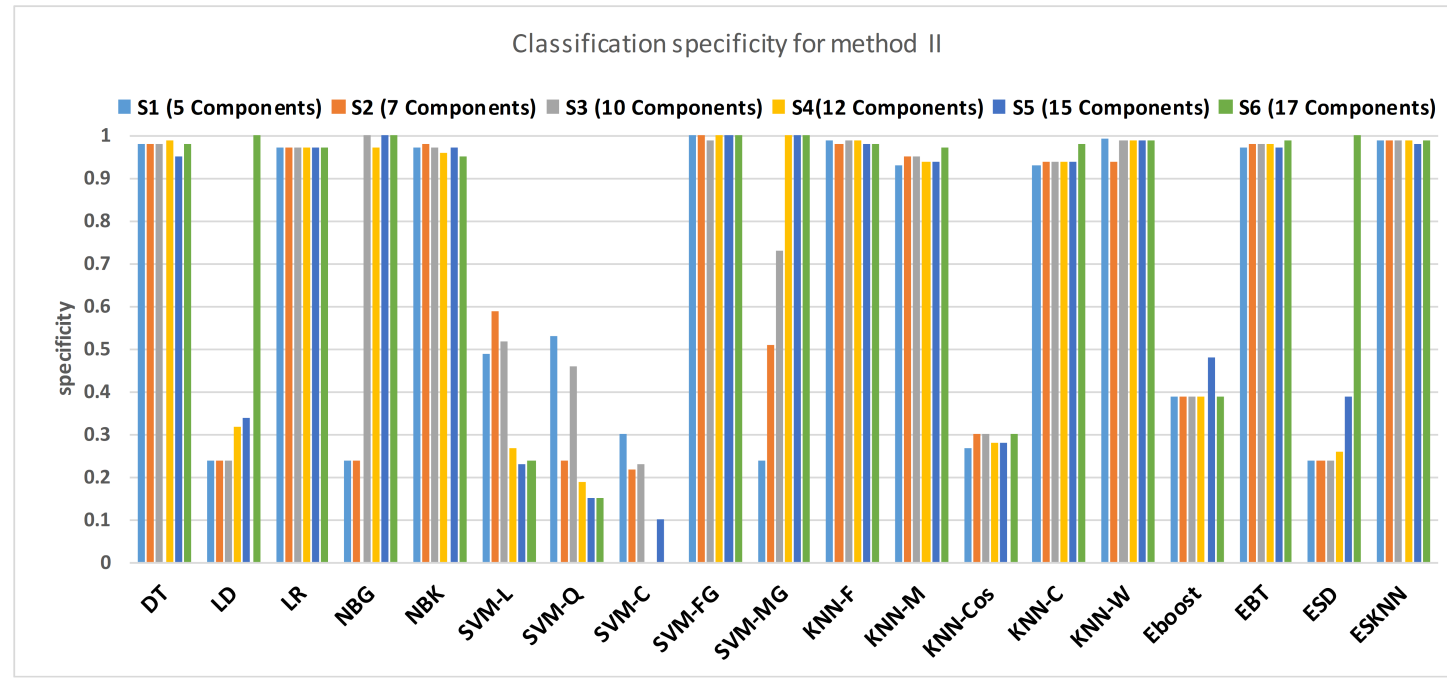

Figure 17. Performance of specificity for different feature sets in various classifiers for PuPG signal through method II.

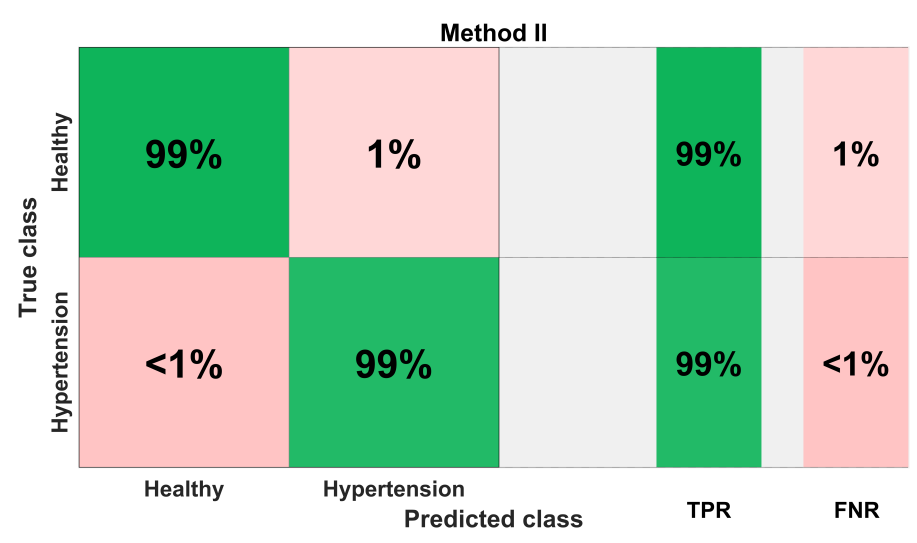

(a) Confusion matrix in terms of percentage

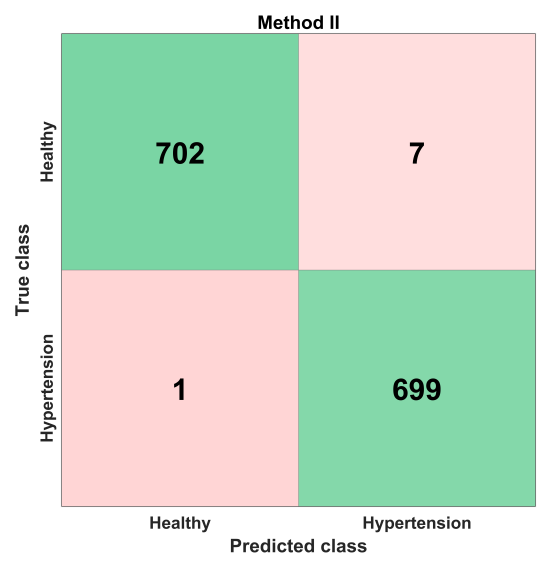

(b) Confusion matrix in terms of numbers

Figure 18. Confusion matrix for method II.

Table 14 includes the results of comprehensive experimentation which is performed to avoid the classifier overfitting. The selected framework was examined through 5-, 10-, $15-$, and 20 -fold cross-validation and $20 \%$ and $25 \%$ train-test holdout validations. For all experimental settings, the proposed scheme achieved more than $98 \%$ accuracy.

Table 14. Validation of the selected scheme of method II.

\begin{tabular}{|c|c|c|c|c|}
\hline Evaluation & Classes & Accuracy & True Positive Rate & False Negative Rate \\
\hline \multirow[b]{2}{*}{5 Fold Cross-Validation } & Healthy & \multirow[b]{2}{*}{0.986} & 0.99 & 0.01 \\
\hline & Hypertension & & 0.98 & 0.02 \\
\hline \multirow[b]{2}{*}{10 Fold Cross-Validation } & Healthy & \multirow[b]{2}{*}{0.994} & 0.99 & 0.01 \\
\hline & Hypertension & & $>0.99$ & $<0.01$ \\
\hline \multirow{2}{*}{15 Fold Cross-Validation } & Healthy & \multirow[b]{2}{*}{0.994} & 0.99 & 0.01 \\
\hline & Hypertension & & $>0.99$ & $<0.01$ \\
\hline \multirow{2}{*}{20 Fold Cross-Validation } & Healthy & \multirow[b]{2}{*}{0.997} & 0.99 & 0.01 \\
\hline & Hypertension & & 1 & 0 \\
\hline \multirow{2}{*}{ 20\% Hold Out Validation } & Healthy & \multirow[b]{2}{*}{0.986} & 1 & 0 \\
\hline & Hypertension & & 0.97 & 0.03 \\
\hline \multirow{2}{*}{$25 \%$ Hold Out Validation } & Healthy & \multirow[b]{2}{*}{0.989} & 0.98 & 0.02 \\
\hline & Hypertension & & 1 & 0 \\
\hline
\end{tabular}




\subsection{Method I versus Method II: A Comparative Analysis}

This section aims to compare both methods I and II analytically. Based on this comparison, we figure out the best working solution for the detection of hypertension through PuPG signals. Method I comprises of preprocessing of PuPG signals through DWT, followed by feature extraction. Extracted features were subjected to the HFSR scheme and finally classified through Ensemble Subspace KNN. Method II consists of EMD-based signal preprocessing followed by feature extraction. Features were fed to KNN-W classifier for distinguishing normal and hypertension data classes after being reduced through the HFSR approach.

Table 15 shows the performance comparison of methods I and II in terms of average accuracy, sensitivity, specificity, error, and number of features. Method I achieves classification performance of $98.4 \%$ accuracy, $97 \%$ sensitivity, and $99 \%$ specificity using 12 transformed features. Method II obtains $99.4 \%, 99.2 \%$, and $99.6 \%$ results of classification accuracy, sensitivity, and specificity respectively through only five reduced features.

Table 15. Performance comparison of methods I and II.

\begin{tabular}{ccc}
\hline Performance & Method I & Method II \\
\hline Accuracy & $98.40 \%$ & $99.40 \%$ \\
Sensitivity & $97.00 \%$ & $99.20 \%$ \\
Specificity & $99.00 \%$ & $99.60 \%$ \\
Error & $0.02 \%$ & $0.60 \%$ \\
\# of features & 12 & 5 \\
\hline
\end{tabular}

Comparative analysis of both methods establishes that method II outperforms method I in terms of achieving better classification accuracy on a reduced number of features. This might be due to the fact that the accuracy achieved in the case of DWT highly depends on the proper wavelet basis selection [64]. The selection of an appropriate basis is challenging especially for non-stationary data [65]. On the other hand, EMD is a fully data-driven, adaptive, and basis-less transformation [66]. Moreover, the IMF selection process of EMD based on relative energy and mean frequency has assisted the selection of useful discriminative signal characteristics.

Figure 19 presents the finalized EHDS (expert hypertension detection system) based on PuPG signal analysis. EHDS first takes raw PuPG signal as input and performs preprocessing through EMD by rejecting the irrelevant IMFs. Next, only 24 significant features highlighted by the hybrid selection scheme are extracted and reduced through KPCA. The final transformed $1 \times 5$ feature vector is fed to $\mathrm{KNN}-\mathrm{W}$ to distinguish the normal and hypertension data classes. Figure 20 illustrates the classification performance of the proposed EHDS as a function of the number of transformed features. It can be observed that the proposed EHDS achieves the optimum performance on only five transformed features. The classification performance shows no notable improvement with the increase in the number of features. 


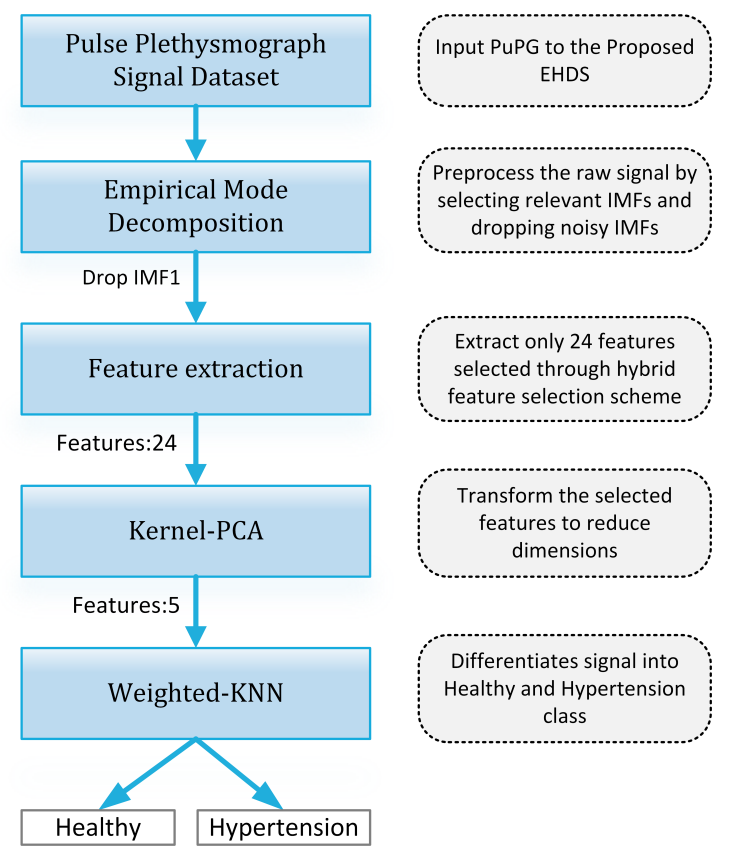

Figure 19. Proposed EHDS block diagram.

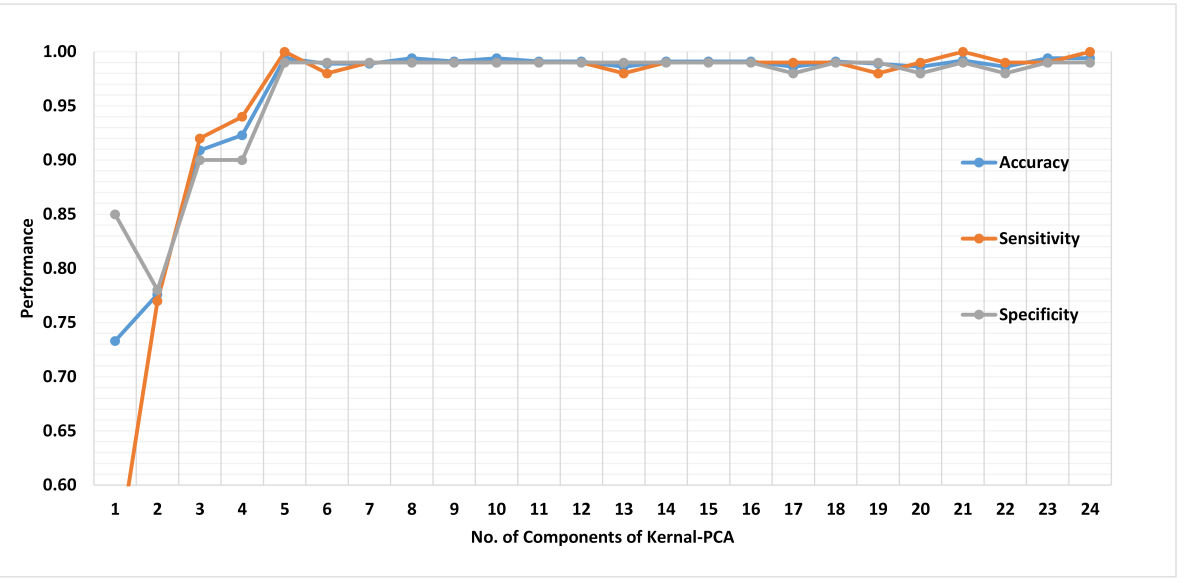

Figure 20. Performance of method II in terms of accuracy, sensitivity, and specificity for 1 to 24 transformed features.

\section{Discussion}

Human blood vessels and the microcirculation system experience transformations with the rise in blood pressure (BP); these changes are exceptionally obvious for patients with severe hypertension. PuPG signals carry a wealth of information about the cardiac health [25-27]. The PuPG signal reflects physical changes in blood volume pressure in blood vessels during the cardiac cycle. The features extracted in this study indicate the changes in Normal and Hypertension PuPG signals acquired from various subjects. The high classification performance of EHDS reflects the association of extracted transformed features with the physiological characteristics of the cardiac condition of the subject. Thus, the proposed expert system may provide a good approximation of the presence or absence of non-communicable diseases such as hypertension.

Table 16 presents a performance comparison of the recent studies. A diagnostic index for the classification of low and high-risk hypertension classes attaining accuracy of $100 \%$ was proposed by [17]. In contrast, our work is targeted towards the classification of Normal and Hypertension classes through PuPG signals. In another study, [18] developed a computational intelligence tool based on ECG signals for the classification of normal and hypertension. EMD was employed in the signal preprocessing stage, followed by nonlinear 
feature extraction from the decomposed IMFs. Extracted features were ranked through Student's $t$-test. The highest classification accuracy of $97.70 \%$ was obtained through the KNN classifier with tenfold cross-validation. A photoplethysmograph (PPG) based detection of hypertension was proposed by [19]. A total of 125 features of various types were extracted and reduced through MRMR. The authors reported the best classification performance with $\mathrm{KNN}-\mathrm{W}$, specifically to be $100 \%, 85.71 \%$, and $92.31 \%$ for positive predictive value, sensitivity, and F1-score, respectively.

Table 16. Comparison with previous works.

\begin{tabular}{|c|c|c|c|c|c|c|c|}
\hline Ref. & Modality & Preprocessing & Features & Feature Reduction & Classification & Data Set & Results \\
\hline [12] & PPG & CWT & GoogLeNet & - & GoogLeNet & MIMIC & F1 score: $92.55 \%$ \\
\hline [13] & $\begin{array}{l}\text { PPG and } \\
\text { ECG }\end{array}$ & - & $\begin{array}{l}\text { PAT and } \\
\text { morphological } \\
\text { features }\end{array}$ & - & KNN & MIMIC & F1 score: $94.84 \%$ \\
\hline [14] & HRV & - & $\begin{array}{l}\text { Standard deviation } \\
\text { of NN intervals }\end{array}$ & - & MIL & $\begin{array}{l}\text { Self-collected data set } \\
\text { Hypertension } 24 \text { and } \\
\text { Normal: } 19\end{array}$ & Accuracy: $85.47 \%$ \\
\hline [15] & ECG & SGF & Entropy features & - & SVM & $\begin{array}{l}\text { Self-collected data set } \\
\text { Hypertension: } 61 \text { and } \\
\text { Normal: } 67\end{array}$ & Accuracy: $93.33 \%$ \\
\hline [16] & HRV & - & $\begin{array}{l}\text { Statistical, spectral, } \\
\text { geometrical, } \\
\text { wavelet, fractal, } \\
\text { and non-linear } \\
\text { features }\end{array}$ & PCA & QDA & $\begin{array}{l}\text { Self-collected data set } \\
\text { Hypertension: } 41 \\
\text { Normal: } 30\end{array}$ & Accuracy: $85.5 \%$ \\
\hline [17] & ECG & OWFB & $\begin{array}{l}\text { Fractal dimension } \\
\text { and } \\
\text { energy features }\end{array}$ & $\begin{array}{l}\text { Student's } \\
t \text {-test }\end{array}$ & $\begin{array}{l}\text { Diagnosis } \\
\text { index }\end{array}$ & $\begin{array}{l}\text { PhysioNet database } \\
\text { High-risk } \\
\text { Hypertension: } 17 \\
\text { subjects } \\
\text { Low-risk Hypertension: } \\
122 \text { subjects } \\
\text { Total: } 139 \text { subjects }\end{array}$ & $\begin{array}{l}100 \% \text { between } \\
\text { low-risk and } \\
\text { high-risk classes }\end{array}$ \\
\hline [18] & ECG & EMD & Entropy features & $\begin{array}{l}\text { Student's } \\
t \text {-test }\end{array}$ & $\begin{array}{l}\text { KNN } \\
\text { classifier }\end{array}$ & $\begin{array}{l}\text { MIT BIH Sinus rhythm } \\
\text { database, SHAREE } \\
\text { database: } \\
\text { Normal: } 18 \text { signals } \\
\text { Hypertension: } 139 \\
\text { signals }\end{array}$ & $\begin{array}{l}\text { Accuracy: } 97.70 \% \\
\text { Sensitivity: } \\
98.90 \% \\
\text { Specificity: } \\
89.10 \%\end{array}$ \\
\hline [19] & PPG & $\begin{array}{l}\text { Chebyshev } \\
\text { II }\end{array}$ & $\begin{array}{l}\text { Time and } \\
\text { morphological } \\
\text { features }\end{array}$ & MRMR & KNN-W & $\begin{array}{l}\text { Hypertension: } 35 \\
\text { Normal: } 48 \\
\text { Total: } 83\end{array}$ & $\begin{array}{l}\text { Positive } \\
\text { Predictive Value: } \\
100 \% \\
\text { Sensitivity: } \\
85.71 \% \\
\text { F1-score: } 92.31 \%\end{array}$ \\
\hline [20] & BCG & & $\begin{array}{l}\text { Morphological } \\
\text { features }\end{array}$ & - & CAR & $\begin{array}{l}\text { Self-collected data set } \\
\text { Hypertension: } 61 \text { and } \\
\text { Normal: } 67\end{array}$ & Accuracy: $84.4 \%$ \\
\hline This study & PuPG & EMD & $\begin{array}{l}\text { Time, frequency, } \\
\text { cepstral, fractal, } \\
\text { and chaotic } \\
\text { features }\end{array}$ & HFSR & KNN-W & $\begin{array}{l}\text { Self-collected data set } \\
\text { Hypertension: } 56 \\
\text { Normal: } 65\end{array}$ & $\begin{array}{l}\text { Accuracy: } 99.7 \% \\
\text { Sensitivity: } 99.2 \% \\
\text { Specificity: } 99.4 \%\end{array}$ \\
\hline
\end{tabular}

The current research is focused on the classification between normal and hypertension data through PuPG signals. To the best of author's knowledge, this is the first study that uses the PuPG signals for discriminating among normal and hypertension with high precision. The current method achieves better performance than the existing ECG- $[15,17,18]$, PPG- [12,19], HRV- [14,16], and BCG-based [20] approaches. Our method also outperforms the fusion-based method for detection of hypertension that utlized a combination of PPG and ECG [13].

The proposed expert system could play a vital role in the early detection of hypertension in low- and middle-income countries. It is important to mention that an estimated 1.04 billion population suffered from hypertension in 2010 [67]. A non-invasive technique based on PuPG signals analysis proposed in this research could be used for the detection of non-communicable diseases. 


\section{Conclusions}

Early detection of hypertension or high blood pressure is extremely significant since it does not cause any obvious symptoms in many people; hence, it can harm the heart, the kidneys, and even the brain. In this study, we proposed an automated detection system for hypertension from PuPG signals for timely and precise screening of disease. First, PuPG signals were preprocessed through EMD, followed by feature extraction of various types. Highly discriminative features were selected through the proposed HFSR scheme that consisted of feature reduction and selection methods. The resultant reduced features of dimension $1 \times 5$ were subjected to various classification methods. The KNN-W classifier achieved the best performance in terms of accuracy, sensitivity, and specificity of $99.4 \%, 99.2 \%$, and $99.6 \%$, respectively. To compute the model performance and avoid overfitting, 5-, 10-, 15-, and 20-fold cross-validations were employed. The proposed method was also compared with the DWT based preprocessing scheme followed by the same feature extraction, selection (HFSR), and classification pipeline. The main advantages of this research are as follows:

- The proposed EHDS system is based on the non-invasive methodology of PuPG signals.

- The EHDS is reliable and less computational intensive with high accuracy.

- The EHDS avoids overfitting as it is validated through 5-, 10-, 15-, and 20-fold cross-validation.

- The proposed approach does not only rely on morphological characteristics of the acquired signal.

- The method can be completely automated, and it works with all qualities of PuPG signals.

Despite the enormous advantages of the proposed method, it has a few limitations.

- The data set used in this research is yet small, with each sample with a length of $10 \mathrm{~s}$.

- The procedure of initial feature extraction and selection of proper IMFs in EMD made the overall process strenuous and time-consuming.

The proposed study conducted a comprehensive comparison of preprocessing schemes (DWT and EMD), feature analysis, selection, and classification as illustrated in Figure 3. The computational complexity of the proposed is significantly low due to the fact that it operates on trained classifier models, therefore eliminating the training computational cost (Figure 19). The proposed system has the potential to be deployed in clinical environments and intensive care units where it can contribute to lessen the workload of medical professionals through its accurate detection and timely diagnosis. In future works, our research group aims to increase the data set size and apply deep learning models to automate the feature extraction process. The proposed framework is intended to be implemented on portable embedded platforms.

Author Contributions: Conceptualization, M.U.K. and S.A.; methodology, M.U.K. and S.A.; software, K.I. and T.A.; validation, F.A. and K.I.; formal analysis, T.A. and K.I.; investigation, K.I. and F.A.; writing-original draft preparation, S.A. and M.U.K.; writing-review and editing, M.A.K. and Y.N.; funding acquisition, Y.N.; project administration, T.A.; data curation, F.A. and M.A.K. All authors have read and agreed to the published version of the manuscript.

Funding: This research was supported by Korea Institute for Advancement of Technology (KIAT) grant funded by the Korea Government (MOTIE) (P0012724, The Competency Development Program for Industry Specialist) and the Soonchunhyang University Research Fund.

Institutional Review Board Statement: Not applicable.

Informed Consent Statement: Informed consent was obtained from all subjects involved in the study.

Data Availability Statement: Data will be available on request. 
Conflicts of Interest: The authors declare no conflict of interest.

\section{References}

1. Tayefi, M.; Esmaeili, H.; Karimian, M.S.; Zadeh, A.A.; Ebrahimi, M.; Safarian, M.; Nematy, M.; Parizadeh, S.M.R.; Ferns, G.A.; Ghayour-Mobarhan, M. The application of a decision tree to establish the parameters associated with hypertension. Comput. Methods Programs Biomed. 2017, 139, 83-91. [CrossRef] [PubMed]

2. WHO. Cardiovascular Diseases. 2017. Available online: https://www.who.int/news-room/fact-sheets/detail/cardiovasculardiseases-(cvds) (accessed on 23 October 2020).

3. WHO. Hypertension, 13 September 2019. Available online: https://www.who.int/news-room/fact-sheets/detail/hypertension (accessed on 23 October 2020).

4. Poddar, M.; Kumar, V.; Sharma, Y.P. Heart rate variability based classification of normal and hypertension cases by linear-nonlinear method. Def. Sci. J. 2014, 64, 542. [CrossRef]

5. Li, W.; Gu, H.; Teo, K.K.; Bo, J.; Wang, Y.; Yang, J.; Wang, X.; Zhang, H.; Sun, Y.; Jia, X.; et al. Hypertension prevalence, awareness, treatment, and control in 115 rural and urban communities involving 47,000 people from China. J. Hypertens. 2016, 34, 39-46. [CrossRef] [PubMed]

6. Wall, H.K.; Hannan, J.A.; Wright, J.S. Patients with undiagnosed hypertension: Hiding in plain sight. JAMA 2014, 312, 1973-1974. [CrossRef] [PubMed]

7. Saleem, F.; Hassali, A.A.; Shafie, A.A. Hypertension in Pakistan: Time to take some serious action. Br. J. Gen. Pract. 2010, 60, 449-450. [CrossRef] [PubMed]

8. Benjamin, E.J.; Blaha, M.J.; Chiuve, S.E.; Cushman, M.; Das, S.R.; Deo, R.; De Ferranti, S.D.; Floyd, J.; Fornage, M.; Gillespie, C.; et al. Heart disease and stroke statistics-2017 update. Circulation 2017, 135, e146-e603. [CrossRef]

9. Goodhart, A.K. Hypertension from the patient's perspective. Br. J. Gen. Pract. 2016, 66, 570. [CrossRef]

10. Huang, C.; Wang, Y.; Li, X.; Ren, L.; Zhao, J.; Hu, Y.; Zhang, L.; Fan, G.; Xu, J.; Gu, X.; et al. Clinical features of patients infected with 2019 novel coronavirus in Wuhan, China. Lancet 2020, 395, 497-506. [CrossRef]

11. Schiffrin, E.L.; Flack, J.M.; Ito, S.; Muntner, P.; Webb, R.C. Hypertension and COVID-19. Am. J. Hypertens. 2020, 33, 373-374. [CrossRef]

12. Liang, Y.; Chen, Z.; Ward, R.; Elgendi, M.J.B. Photoplethysmography and deep learning: Enhancing hypertension risk stratification. Biosensors 2018, 8, 101. [CrossRef]

13. Liang, Y.; Chen, Z.; Ward, R.; Elgendi, M. Hypertension assessment via ECG and PPG signals: An evaluation using MIMIC database. Diagnostics 2018, 8, 65. [CrossRef] [PubMed]

14. Lan, K.c.; Raknim, P.; Kao, W.F.; Huang, J.H. Toward hypertension prediction based on PPG-derived HRV signals: A feasibility study. J. Med. Syst. 2018, 42, 103. [CrossRef] [PubMed]

15. Ni, H.; Cho, S.; Mankoff, J.; Yang, J.J.; Computing, H. Automated recognition of hypertension through overnight continuous HRV monitoring. J. Ambient. Intell. Humaniz. Comput. 2018, 9, 2011-2023. [CrossRef]

16. Kublanov, V.S.; Dolganov, A.Y.; Belo, D.; Gamboa, H.J. Comparison of machine learning methods for the arterial hypertension diagnostics. Appl. Bionics Biomech. 2017, 2017, 5985479. [CrossRef] [PubMed]

17. Rajput, J.S.; Sharma, M.; Acharya, U.R. Hypertension Diagnosis Index for Discrimination of High-Risk Hypertension ECG Signals Using Optimal Orthogonal Wavelet Filter Bank. Int. J. Environ. Res. Public Health 2019, 16, 4068. [CrossRef]

18. Soh, D.C.K.; Ng, E.; Jahmunah, V.; Oh, S.L.; San, T.R.; Acharya, U.R. A computational intelligence tool for the detection of hypertension using empirical mode decomposition. Medicine 2020, 118, 103630. [CrossRef]

19. Liang, Y.; Chen, Z.; Ward, R.; Elgendi, M. Hypertension assessment using photoplethysmography: A risk stratification approach. J. Clin. Med. 2019, 8, 12. [CrossRef]

20. Liu, F.; Zhou, X.; Wang, Z.; Cao, J.; Wang, H.; Zhang, Y. Unobtrusive mattress-based identification of hypertension by integrating classification and association rule mining. Sensors 2019, 19, 1489. [CrossRef]

21. Baranchuk, A.; Kang, J.; Shaw, C.; Campbell, D.; Ribas, S.; Hopman, W.M.; Alanazi, H.; Redfearn, D.P.; Simpson, C.S. Electromagnetic interference of communication devices on ECG machines. Clin. Cardiol. Int. Index. Peer Rev. J. Adv. Treat. Cardiovasc. Dis. 2009, 32, 588-592. [CrossRef]

22. Klein, A.; Djaiani, G. Mobile phones in the hospital-past, present and future. Anaesthesia 2003, 58, 353-357. [CrossRef]

23. Kranjec, J.; Beguš, S.; Geršak, G.; Drnovšek, J. Non-contact heart rate and heart rate variability measurements: A review. Biomed. Signal Process. Control. 2014, 13, 102-112. [CrossRef]

24. Ave, A.; Fauzan, H.; Adhitya, S.R.; Zakaria, H. Early detection of cardiovascular disease with photoplethysmogram (PPG) sensor. In Proceedings of the 2015 International Conference on Electrical Engineering and Informatics (ICEEI), Denpasar, Indonesia, 10-11 August 2015; pp. 676-681.

25. Khan, M.U.; Aziz, S.; Malik, A.; Imtiaz, M.A. Detection of Myocardial Infarction using Pulse Plethysmograph Signals. In Proceedings of the 2019 International Conference on Frontiers of Information Technology (FIT), Islamabad, Pakistan, 16-18 December 2019; pp. 95-955.

26. Khan, M.U.; Aziz, S.; Amjad, F.; Mohsin, M. Detection of Dilated Cardiomyopathy using Pulse Plethysmographic Signal Analysis. In Proceedings of the 2019 22nd International Multitopic Conference (INMIC), Islamabad, Pakistan, 29-30 November 2019; pp. 1-5. 
27. Khan, M.U.; Aziz, S.; Iqtidar, K.; Zainab, A.; Saud, A. Prediction of Acute Coronary Syndrome Using Pulse Plethysmograph. In Proceedings of the 2019 4th International Conference on Emerging Trends in Engineering, Sciences and Technology (ICEEST), Karachi, Pakistan, 10-11 December 2019; pp. 1-6.

28. Naqvi, S.Z.H.; Aziz, S.; Khan, M.U.; Asghar, N.; Rasool, G. Emotion Recognition System using Pulse Plethysmograph. In Proceedings of the 2020 International Conference on Emerging Trends in Smart Technologies (ICETST), Karachi, Pakistan, 2627 March 2020; pp. 1-6.

29. Khan, M.U.; Aziz, S.; Naqvi, S.Z.H.; Zaib, A.; Maqsood, A. Pattern Analysis Towards Human Verification using Photoplethysmograph Signals. In Proceedings of the 2020 International Conference on Emerging Trends in Smart Technologies (ICETST), Karachi, Pakistan, 26-27 March 2020; pp. 1-6.

30. Zhang, G.; Si, Y.; Yang, W.; Wang, D. A Robust Multilevel DWT Densely Network for Cardiovascular Disease Classification. Sensors 2020, 20, 4777. [CrossRef] [PubMed]

31. Aileni, R.M.; Pasca, S.; Florescu, A. EEG-Brain Activity Monitoring and Predictive Analysis of Signals Using Artificial Neural Networks. Sensors 2020, 20, 3346. [CrossRef] [PubMed]

32. Alturki, F.A.; AlSharabi, K.; Abdurraqeeb, A.M.; Aljalal, M. EEG Signal Analysis for Diagnosing Neurological Disorders Using Discrete Wavelet Transform and Intelligent Techniques. Sensors 2020, 20, 2505. [CrossRef] [PubMed]

33. Gradolewski, D.; Redlarski, G. Wavelet-based denoising method for real phonocardiography signal recorded by mobile devices in noisy environment. Comput. Biol. Med. 2014, 52, 119-129. [CrossRef] [PubMed]

34. Ebrahim, M.P.; Heydari, F.; Redoute, J.M.; Yuce, M.R. Accurate heart rate detection from on-body continuous wave radar sensors using wavelet transform. In Proceedings of the 2018 IEEE SENSORS, New Delhi, India, 28-31 October 2018; pp. 1-4.

35. Ebrahim, M.P.; Heydari, F.; Walker, K.; Joe, K.; Redoute, J.M.; Yuce, M.R. Systolic Blood Pressure Estimation Using Wearable Radar and Photoplethysmogram Signals. In Proceedings of the 2019 IEEE International Conference on Systems, Man and Cybernetics (SMC), Bari, Italy, 6-9 October 2019; pp. 3878-3882.

36. Asghar, M.A.; Khan, M.J.; Rizwan, M.; Mehmood, R.M.; Kim, S.H. An Innovative Multi-Model Neural Network Approach for Feature Selection in Emotion Recognition Using Deep Feature Clustering. Sensors 2020, 20, 3765. [CrossRef] [PubMed]

37. Aziz, S.; Khan, M.U.; Alhaisoni, M.; Akram, T.; Altaf, M. Phonocardiogram Signal Processing for Automatic Diagnosis of Congenital Heart Disorders through Fusion of Temporal and Cepstral Features. Sensors 2020, 20, 3790. [CrossRef]

38. Tsimpiris, A.; Kugiumtzis, D. Feature selection for classification of oscillating time series. Expert Syst. 2012, 29 , 456-477. [CrossRef]

39. Goyal, D.; Choudhary, A.; Pabla, B.; Dhami, S. Support vector machines based non-contact fault diagnosis system for bearings. J. Intell. Manuf. 2019, 31, 1275-1289. [CrossRef]

40. Fasil, O.; Rajesh, R. Time-domain exponential energy for epileptic EEG signal classification. Neurosci. Lett. 2019, $694,1-8$.

41. Banker, R.; Natarajan, R.; Zhang, D. Two-stage estimation of the impact of contextual variables in stochastic frontier production function models using data envelopment analysis: Second stage OLS versus bootstrap approaches. Eur. J. Oper. Res. 2019, 278, 368-384. [CrossRef]

42. Kumar, D.; Carvalho, P.; Antunes, M.; Paiva, R.; Henriques, J. Heart murmur classification with feature selection. In Proceedings of the 2010 Annual International Conference of the IEEE Engineering in Medicine and Biology, Buenos Aires, Argentina, 31 August-4 September 2010; pp. 4566-4569.

43. Dhindsa, I.S.; Agarwal, R.; Ryait, H.S. Performance evaluation of various classifiers for predicting knee angle from electromyography signals. Expert Syst. 2019, 36, e12381. [CrossRef]

44. Mukhopadhyay, A.K.; Samui, S. An experimental study on upper limb position invariant EMG signal classification based on deep neural network. Biomed. Signal Process. Control. 2020, 55, 101669. [CrossRef]

45. Phinyomark, A.; Phukpattaranont, P.; Limsakul, C. Feature reduction and selection for EMG signal classification. Expert Syst. Appl. 2012, 39, 7420-7431. [CrossRef]

46. Peeters, G. A large set of audio features for sound description (similarity and classification) in the CUIDADO project. Cuid. IST Proj. Rep. 2004, 54, 1-25.

47. Yadav, A.; Singh, A.; Dutta, M.K.; Travieso, C.M. Machine learning-based classification of cardiac diseases from PCG recorded heart sounds. Neural Comput. Appl. 2019,1-14. [CrossRef]

48. Amezquita-Sanchez, J.P.; Mammone, N.; Morabito, F.C.; Marino, S.; Adeli, H. A novel methodology for automated differential diagnosis of mild cognitive impairment and the Alzheimer's disease using EEG signals. J. Neurosci. Methods 2019, 322, 88-95. [CrossRef]

49. Peng, J.; Hao, D.; Yang, L.; Du, M.; Song, X.; Jiang, H.; Zhang, Y.; Zheng, D. Evaluation of electrohysterogram measured from different gestational weeks for recognizing preterm delivery: A preliminary study using random Forest. Biocybern. Biomed. Eng. 2020, 40, 352-362. [CrossRef]

50. Birajdar, G.K.; Patil, M.D. Speech/music classification using visual and spectral chromagram features. J. Ambient. Intell. Humaniz. Comput. 2020, 11, 329-347. [CrossRef]

51. Leite, J.P.R.; Moreno, R.L. Heartbeat classification with low computational cost using Hjorth parameters. IET Signal Process. 2017, 12, 431-438. [CrossRef]

52. Dua, M.; Aggarwal, R.K.; Biswas, M. GFCC based discriminatively trained noise robust continuous ASR system for Hindi language. J. Ambient. Intell. Humaniz. Comput. 2019, 10, 2301-2314. [CrossRef] 
53. Adnan, S.M.; Irtaza, A.; Aziz, S.; Ullah, M.O.; Javed, A.; Mahmood, M.T. Fall detection through acoustic local ternary patterns. Appl. Acoust. 2018, 140, 296-300. [CrossRef]

54. Guyon, I. Practical feature selection: From correlation to causality. In Proceedings of the NATO Advanced Study Institute on Mining Massive Data Sets for Security (MMDSS 2007), Gazzada, Italy, 10-21 September 2007; pp. 27-43.

55. Li, X.; Ling, S.H.; Su, S. A Hybrid Feature Selection and Extraction Methods for Sleep Apnea Detection Using Bio-Signals. Sensors 2020, 20, 4323. [CrossRef]

56. Zhen, Z.; Zeng, X.; Wang, H.; Han, L. A global evaluation criterion for feature selection in text categorization using KullbackLeibler divergence. In Proceedings of the 2011 International Conference of Soft Computing and Pattern Recognition (SoCPaR), Dalian, China, 14-16 October 2011; pp. 440-445.

57. Guorong, X.; Peiqi, C.; Minhui, W. Bhattacharyya distance feature selection. In Proceedings of the 13th International Conference on Pattern Recognition, Vienna, Austria, 25-29 August 1996; Volume 2, pp. 195-199.

58. Chowdhury, M.H.; Shuzan, M.N.I.; Chowdhury, M.E.; Mahbub, Z.B.; Uddin, M.M.; Khandakar, A.; Reaz, M.B.I. Estimating Blood Pressure from the Photoplethysmogram Signal and Demographic Features Using Machine Learning Techniques. Sensors 2020, 20, 3127. [CrossRef] [PubMed]

59. Ding, C.; Peng, H. Minimum redundancy feature selection from microarray gene expression data. J. Bioinform. Comput. Biol. 2005, 3, 185-205. [CrossRef] [PubMed]

60. Xu, Y.; Zhao, X.; Chen, Y.; Zhao, W. Research on a mixed gas recognition and concentration detection algorithm based on a metal oxide semiconductor olfactory system sensor array. Sensors 2018, 18, 3264. [CrossRef] [PubMed]

61. Schölkopf, B.; Smola, A.; Müller, K.R. Nonlinear component analysis as a kernel eigenvalue problem. Neural Comput. 1998, 10, 1299-1319. [CrossRef]

62. Khan, M.U.; Saad, M.; Aziz, S.; Ch, J.M.; Naqvi. S.Z.H.; Qasim, M.A. Electrocardiogram based Gender Classification. In Proceedings of the 2020 International Conference on Electrical, Communication, and Computer Engineering (ICECCE), Istanbul, Turkey, 12-13 June 2020; pp. 1-6.

63. Hoffmann, H. Kernel PCA for novelty detection. Pattern Recognit. 2007, 40, 863-874. [CrossRef]

64. Mohamed, M.; Deriche, M. An approach for ECG feature extraction using daubechies 4 (DB4) wavelet. Int. J. Comput. Appl. 2014, $96,36-41$.

65. Acharya, U.R.; Fujita, H.; Adam, M.; Lih, O.S.; Sudarshan, V.K.; Hong, T.J.; Koh, J.E.; Hagiwara, Y.; Chua, C.K.; Poo, C.K.; et al. Automated characterization and classification of coronary artery disease and myocardial infarction by decomposition of ECG signals: A comparative study. Inf. Sci. 2017, 377, 17-29. [CrossRef]

66. Labate, D.; La Foresta, F.; Occhiuto, G.; Morabito, F.C.; Lay-Ekuakille, A.; Vergallo, P. Empirical mode decomposition vs. wavelet decomposition for the extraction of respiratory signal from single-channel ECG: A comparison. IEEE Sens. J. 2013, 13, 2666-2674. [CrossRef]

67. Mills, K.T.; Bundy, J.D.; Kelly, T.N.; Reed, J.E.; Kearney, P.M.; Reynolds, K.; Chen, J.; He, J. Global disparities of hypertension prevalence and control: A systematic analysis of population-based studies from 90 countries. Circulation 2016, 134, 441-450. [CrossRef] [PubMed] 